\title{
Towards a Complete Classification of Symmetry-Protected Topological Phases for Interacting Fermions in Three Dimensions and a General Group Supercohomology Theory
}

\author{
Qing-Rui Wang and Zheng-Cheng Gu* \\ Department of Physics, The Chinese University of Hong Kong, Shatin, New Territories, Hong Kong, China
}

(Received 8 August 2017; published 30 March 2018)

\begin{abstract}
The classification and construction of symmetry-protected topological (SPT) phases in interacting boson and fermion systems have become a fascinating theoretical direction in recent years. It has been shown that (generalized) group cohomology theory or cobordism theory gives rise to a complete classification of SPT phases in interacting boson or spin systems. The construction and classification of SPT phases in interacting fermion systems are much more complicated, especially in three dimensions. In this work, we revisit this problem based on an equivalence class of fermionic symmetric local unitary transformations. We construct very general fixed-point SPT wave functions for interacting fermion systems. We naturally reproduce the partial classifications given by special group supercohomology theory, and we show that with an additional $\tilde{B} H^{2}\left(G_{b}, \mathbb{Z}_{2}\right)$ structure [the so-called obstruction-free subgroup of $H^{2}\left(G_{b}, \mathbb{Z}_{2}\right)$ ], a complete classification of SPT phases for three-dimensional interacting fermion systems with a total symmetry group $G_{f}=G_{b} \times \mathbb{Z}_{2}^{f}$ can be obtained for unitary symmetry group $G_{b}$. We also discuss the procedure for deriving a general group supercohomology theory in arbitrary dimensions.
\end{abstract}

DOI: 10.1103/PhysRevX.8.011055

Subject Areas: Condensed Matter Physics,

Quantum Information,

Strongly Correlated Materials

\section{INTRODUCTION}

In recent years, a new type of topological ordersymmetry-protected topological (SPT) order [1-3]—has been proposed and intensively studied in interacting boson and fermion systems. Two-dimensional and three-dimensional topological insulators (TIs) [4,5] are the simplest examples of SPT phases, which are protected by timereversal and charge-conservation symmetries. Although TIs were initially proposed and experimentally realized in essentially noninteracting electron systems, very recent studies have established their existence and stability even in the presence of strong interactions, by identifying nonperturbative quantum anomalies on various manifolds [6]. The first attempt to systematically understand SPT phases in interacting systems was proposed in Ref. [1], in which the author pointed out that the well-known spin-1 Haldane chain [7] was actually an SPT phase. Later, a systematic classification of SPT phases for interacting bosonic systems in arbitrary dimensions with arbitrary global symmetry was

\footnotetext{
*zcgu@phy.cuhk.edu.hk
}

Published by the American Physical Society under the terms of the Creative Commons Attribution 4.0 International license. Further distribution of this work must maintain attribution to the author(s) and the published article's title, journal citation, and DOI. achieved using generalized group cohomology theory $[2,3,8]$ or cobordism theory [9]. This systematic classification essentially classifies the quantum anomalies associated with the corresponding global symmetries in interacting bosonic systems. In terms of the physical picture, it has been further pointed out that by gauging the global symmetry $G$, different SPT phases can be characterized by different types of braiding statistics of $G$-flux or flux lines in 2D or 3D [1017]. Anomalous surface topological order has also been proposed as another very powerful way to identify and characterize different 3D SPT phases in interacting boson and fermion systems [18-27].

From the quantum information perspective, intrinsic topological phases are gapped quantum states that can be defined and classified by an equivalence class of finite depth local unitary transformations [28], which leads to the novel concept of long-range entanglement. However, in contrast to those intrinsic topological phases, all SPT phases can be adiabatically connected to a trivial disordered phase or to an atomic insulator without symmetry protection. Therefore, SPT phases contain only short-range entanglement, and can be constructed by applying local unitary transformations on a trivial product state. In particular, Refs. [2,3] introduced a systematic way of constructing fixed-point ground-state wave functions for bosonic SPT phases on arbitrary triangulations in arbitrary dimensions. Such a construction is fairly complete for 
bosonic SPT phases protected by unitary symmetry groups (up to 3D). So far, the only known example beyond this construction is the so-called efm $f$ SPT state [18], which is protected by time-reversal symmetry in 3D. Later, it was shown that such an exotic bosonic SPT state could be realized by the Walker-Wang model $[29,30]$.

The classification and systematic understanding of SPT phases in interacting fermion systems are much more complicated. One obvious way to achieve fruitful results is to study the reduction of the free-fermion classifications [31,32] under the effect of interactions [33-39]. However, this approach misses those fermionic SPT (FSPT) phases that cannot be realized in free-fermion systems $[12,40]$. A slightly general way to understand FSPT in interacting fermion systems is to stack some additional bosonic SPT states onto a free-fermion SPT state [26]. An arguably fairly complete classification of TIs in interacting electron systems [41] can be constructed in such a way. However, there is no natural reason to believe that all FSPT phases in interacting systems can be realized by the abovementioned stacking constructions, and counterexamples can be constructed explicitly. Moreover, it has been shown that certain bosonic SPT phases become "trivial" (adiabatically connected to a product state) $[26,42]$ when embedded into interacting fermion systems. Apparently, the stacking construction cannot explain all of these subtle issues. Therefore, a systematic understanding and the construction of interacting FSPT phases are very desirable.

The first attempt to classify interacting FSPT phases in general dimensions was proposed in Ref. [42], in which a class of FSPT phases was constructed systematically by generalizing the usual group cohomology theory into the so-called special group supercohomology theory. However, it turns out that such a construction cannot give rise to all FSPT phases except in one dimension, where the obtained classification of FSPT phases perfectly agrees with previous results $[43,44])$. On the other hand, quantum anomalies characterized by spin cobordism [45] or invertible spin topological quantum field theory (TQFT) [46-48] suggest a rich diversity of FSPT phases, although it is not clear how to construct these FSPT states in an explicit and systematic way.

Alternatively, the idea of gauging fermion parity [37,49-52] provides another way to understand FSPT. In 2D, a fairly complete classification of FSPT can be obtained in this way, which also agrees with the anomaly classification given by spin cobordism and invertible spin TQFT $[45,46,48,53,54]$. It has been shown that the mathematical objects that classify 2D FSPT phases with a total symmetry $G_{f}=G_{b} \times \mathbb{Z}_{2}^{f}$ (where $G_{b}$ is the bosonic global symmetry and $\mathbb{Z}_{2}^{f}$ is the fermion parity conservation symmetry) can be summarized as three group cohomologies of the symmetry group $G_{b}[48,50]$ : $H^{1}\left(G_{b}, \mathbb{Z}_{2}\right), \quad B H^{2}\left(G_{b}, \mathbb{Z}_{2}\right), \quad$ and $\quad H^{3}\left(G_{b}, U_{T}(1)\right)$. $H^{1}\left(G_{b}, \mathbb{Z}_{2}\right)$, which corresponds one to one to the $\mathbb{Z}_{2}$ subgroups of $G_{b}$, classifies FSPT phases with Majorana edge modes. $B H^{2}\left(G_{b}, \mathbb{Z}_{2}\right)$, the obstruction-free subgroup of $H^{2}\left(G_{b}, \mathbb{Z}_{2}\right)$, is formed by elements $n_{2} \in$ $H^{2}\left(G_{b}, \mathbb{Z}_{2}\right)$ that satisfy $S q^{2}\left(n_{2}\right)=0$ in $H^{4}\left(G_{b}, U_{T}(1)\right)$, where $S q^{2}$ is the Steenrod square, $S q^{2}: H^{d}\left(G_{b}, \mathbb{Z}_{2}\right) \rightarrow$ $H^{d+2}\left(G_{b}, \mathbb{Z}_{2}\right) \subset H^{d+2}\left[G_{b}, U_{T}(1)\right] . H^{3}\left(G_{b}, U_{T}(1)\right)$ is the well-known classification of bosonic SPT phases. Physically, the $H^{1}\left(G_{b}, \mathbb{Z}_{2}\right)$ layer can be constructed by decorating a Majorana chain [55], which is a one-dimensional invertible fermionic TQFT, onto the domain walls of symmetry group $G_{b}$. The $B H^{2}\left(G_{b}, \mathbb{Z}_{2}\right)$ layer can be constructed by decorating complex fermions, which are zero-dimensional invertible TQFT, onto the intersection points of $G_{b}$-symmetry domain walls. Nevertheless, the decoration scheme can suffer from obstructions, and only subgroup $B H^{2}\left(G_{b}, \mathbb{Z}_{2}\right)$ classifies valid and inequivalent $2 \mathrm{D}$ FSPT phases. Some interesting examples of SPT phases have been studied in 3D based on a Walker-Wang model construction [56], e.g., DIII-class topological superconductors [24,57-59]. Unfortunately, it is impossible to construct all FSPT phases using the Walker-Wang model. It is even unclear how to reproduce all of the special group supercohomology constructions in this way. Very recently, some new interacting FSPT phases beyond special group supercohomology were formally proposed by using spin TQFT [60]. However, a general principle and lattice model realization are still lacking.

\section{A. Classify FSPT phases via equivalence classes of fermionic symmetric local unitary transformations}

In this paper, we propose a general physical principle to construct all FSPT phases in 3D with the total symmetry group $G_{f}=G_{b} \times \mathbb{Z}_{2}^{f}$. A previous work showed that in the presence of global symmetry, symmetry-enriched topological (SET) phases can be defined and classified by equivalence classes of symmetric local unitary transformations $[61,62]$. In particular, SPT phases can be realized as a special class of SET phases whose bulk excitations are trivial and can be adiabatically connected to a product state in the absence of global symmetry.

In Ref. [63], it was shown that fermionic local unitary (FLU) transformations can be used to define and classify intrinsic topological phases for interacting fermion systems. The Fock space structure and fermion parity conservation symmetry of fermion systems can be naturally encoded into FLU transformations. Let us first briefly review the definition of FLU transformation. Similar to the local bosonic systems, the finite-time evolution generated by a local fermion Hamiltonian defines an equivalence relation between gapped states in interacting fermion systems:

$|\psi(1)\rangle \sim|\psi(0)\rangle$ iff $|\psi(1)\rangle=\mathcal{T}\left[e^{-i \int_{0}^{1} d \lambda \tilde{H}_{f}(\lambda)}\right]|\psi(0)\rangle$, 
where $\mathcal{T}$ is the path-ordering operator and $\tilde{H}_{f}(\lambda)$ is a local fermionic Hamiltonian defined in Fock space. We call $\mathcal{T}\left[e^{-i \int_{0}^{1} d \lambda \tilde{H}_{f}(\lambda)}\right]$ an FLU evolution. It is well known that the finite-time FLU evolution is closely related to fermionic quantum circuits with finite depth, which is defined through piecewise FLU operators. A piecewise FLU operator has the form $U_{\mathrm{pwl}}=\prod_{i} e^{-i H_{f}(\boldsymbol{i})} \equiv \prod_{i} U(\boldsymbol{i})$, where $H_{f}(\boldsymbol{i})$ is a fermionic Hermitian operator and $U(\boldsymbol{i})$ is the corresponding fermionic unitary operator defined in Fock space that preserves fermion parity (e.g., contains even number of fermion creation and annihilation operators) and acts on a region labeled by $i$. Note that regions labeled by different $\boldsymbol{i}$ 's are not overlapping. We further require that the size of each region is less than some finite number $l$. The unitary operator $U_{\text {pwl }}$ defined in this way is called a piecewise fermionic local unitary operator with range $l$. A fermion quantum circuit with depth $M$ is given by the product of $M$ piecewise fermionic local unitary operators: $U_{\text {circ }}^{M}=U_{\mathrm{pwl}}^{(1)} U_{\mathrm{pwl}}^{(2)} \ldots U_{\mathrm{pwl}}^{(M)}$. It is believed that any FLU evolution can be simulated with a constant depth fermionic quantum circuit and vice versa. Therefore, the equivalence relation between gapped states in interacting fermion systems can be rewritten in terms of constant depth fermionic quantum circuits:

$$
|\psi(1)\rangle \sim|\psi(0)\rangle \text { iff }|\psi(1)\rangle=U_{\text {circ }}^{M}|\psi(0)\rangle .
$$

Thus, we can use the term FLU transformation to refer to both FLU evolution and constant depth fermionic quantum circuit. From the definition of the FSPT state, it is easy to see that (in the absence of global symmetry)

$$
\left.|\mathrm{FSPT}\rangle=U_{\text {circ }}^{M} \mid \text { Trivial }\right\rangle .
$$

Namely, an FSPT state can be connected to a trivial state (e.g., a product state) vial FLU transformation (in the absence of global symmetry). Now let us consider the entanglement density matrix $\rho_{A}$ of an FSPT state in region $A$. $\rho_{A}$ may act on a subspace of the Hilbert space in region $A$, and the subspace is called the support space $\tilde{V}_{A}$ of region $A$. Clearly, Eq. (3) implies that the support space of any FSPT in region $A$ must be one dimensional. This is simply because a trivial state (e.g., a product state) has a onedimensional support space, and any FSPT state will become a product state via a proper local basis change (induced by an FLU transformation).

In the presence of global symmetry, we can further introduce the notion of fermionic symmetric local unitary (FSLU) transformations to define and classify fermionic SET (FSET) phases in interacting fermion systems. By FSLU transformation, we mean the corresponding piecewise FLU operator is invariant under symmetry $G_{b}$. More precisely, we have $\quad U_{\mathrm{pwl}}=\prod_{i} e^{-i H_{f}\left(g_{i 0}, g_{i 1}, g_{i 2}, \ldots\right)} \equiv \prod_{i} U\left(g_{i 0}, g_{i}, g_{i 2}, \ldots\right)$ and $U\left(g g_{i 0}, g g_{i 2}, g g_{i 3}, \ldots\right)=U\left(g_{i 0}, g_{i 1}, g_{i 2}, \ldots\right)$ for any $g \in G_{b}$. (We note that here we choose the group element basis $g_{i 0}, g_{i 1}, g_{i 2}, \ldots$ to represent a fermionic symmetric unitary operator acting on a region labeled by $i$.) Again, FSPT phases are a special class of FSET phases that have trivial bulk excitation and can be adiabatically connected to a product state in the absence of global symmetry. Thus, we only need to enforce the FSLU transformations to be one dimensional (when acting on the support space $\rho_{A}$ for any region $A$ ) to classify all FSPT states.

\section{B. Summary of main results}

It turns out that the novel concept of FSLU transformation allows us to construct very general fixed-point FSPT states of 2D and 3D FSPT phases. All of these fixedpoint wave functions admit exactly solvable parent Hamiltonians consisting of commuting projectors on an arbitrary triangulation with an arbitrary branching structure. We begin with the 2D case, in which the discrete spin structure can be implemented by Kasteleyn orientations [64-66], allowing us to decorate Majorana chains onto $G_{b^{-}}$ symmetry domain walls $[67,68]$. We then show how to implement the discrete spin structure on a triangulation of a 3D orientable spin manifold, which is a nontrivial generalization of 2D Kasteleyn orientation. The discrete spin structure allows us to decorate the Majorana chains onto the intersection lines of $G_{b}$-symmetry domain walls in a selfconsistent and topologically invariant way. The fundamental mathematical data describing such a decoration scheme belong to $H^{2}\left(G_{b}, \mathbb{Z}_{2}\right)$, subjected to an obstruction on $H^{4}\left(G_{b}, \mathbb{Z}_{2}\right)$. The obstruction can be understood through the following physical picture. As Kasteleyn orientation is not always possible for a large loop (the 3D discrete spin structure can be used to construct local Kasteleyn orientations of small loops), complex fermion decoration on the intersection points of $G_{b}$-symmetry domain walls is typically required, and this is only possible when the $H^{4}\left(G_{b}, \mathbb{Z}_{2}\right)$ obstruction vanishes. Furthermore, another obstruction on $H^{5}\left(G_{b}, U_{T}(1)\right)$ is generated by wave function renormalization to finally determine whether the entire decoration scheme of Majorana chains is valid for a fixed-point wave function in 3D.

The precise mathematical objects that classify 3D FSPT phases with a total symmetry $G_{f}=G_{b} \times \mathbb{Z}_{2}^{f}$ can also be summarized as three group cohomologies of the symmetry group $G_{b}: \tilde{B} H^{2}\left(G_{b}, \mathbb{Z}_{2}\right), B H^{3}\left(G_{b}, \mathbb{Z}_{2}\right)$, and $H_{\text {rigid }}^{4}\left(G_{b}, U_{T}(1)\right) . \tilde{B} H^{2}\left(G_{b}, \mathbb{Z}_{2}\right)$, the obstruction-free subgroup of $H^{2}\left(G_{b}, \mathbb{Z}_{2}\right)$, is formed by elements $\tilde{n}_{2} \in$ $H^{2}\left(G_{b}, \mathbb{Z}_{2}\right)$ that simultaneously satisfy $S q^{2}\left(\tilde{n}_{2}\right)=0$ in $H^{4}\left(G_{b}, \mathbb{Z}_{2}\right)$ and $\mathcal{O}\left(\tilde{n}_{2}\right)=0$ in $H^{5}\left(G_{b}, U_{T}(1)\right)$, where $\mathcal{O}$ is some unknown cohomology operation (to the best of our knowledge) that maps $\tilde{n}_{2}$ satisfying $S q^{2}\left(\tilde{n}_{2}\right)=0$ in $H^{2}\left(G_{b}, \mathbb{Z}_{2}\right)$ into an element in $H^{5}\left(G_{b}, \mathbb{Z}_{8}\right) \subset H^{5}\left[G_{b}, U_{T}(1)\right]$. The explicit expression of $\mathcal{O}$ is very complicated, and it is computed in a physical way 
TABLE I. Classifying FSPT phases up to spacial dimension $d_{\text {sp }}=3$ with a total symmetry $G_{f}=G_{b} \times \mathbb{Z}_{2}^{f}$ using a general group supercohomology class computed from short exact sequences. Note that $\mathcal{H}^{d_{\mathrm{sp}}+1}\left[G_{f}, U_{T}(1)\right]$ is the so-called special group supercohomology proposed in Ref. [42], and that in lower dimensions with $d_{\mathrm{sp}}=0,1$, we have $H_{f}^{d_{\mathrm{sp}}+1}\left[G_{f}, U_{T}(1)\right] \equiv$ $\mathcal{H}^{d_{\mathrm{sp}}+1}\left[G_{f}, U_{T}(1)\right]$.

\begin{tabular}{cc}
\hline \hline$d_{\mathrm{sp}}$ & Short exact sequence \\
\hline 0 & $0 \rightarrow H^{1}\left[G_{b}, U_{T}(1)\right] \rightarrow H_{f}^{1}\left[G_{f}, U_{T}(1)\right] \rightarrow \mathbb{Z}_{2} \rightarrow 0$ \\
1 & $0 \rightarrow H^{2}\left[G_{b}, U_{T}(1)\right] \rightarrow H_{f}^{2}\left[G_{f}, U_{T}(1)\right] \rightarrow H^{1}\left(G_{b}, \mathbb{Z}_{2}\right) \rightarrow 0$ \\
2 & $0 \rightarrow H^{3}\left[G_{b}, U_{T}(1)\right] \rightarrow \mathcal{H}^{3}\left[G_{f}, U_{T}(1)\right] \rightarrow B H^{2}\left(G_{b}, \mathbb{Z}_{2}\right) \rightarrow 0$ \\
& $0 \rightarrow \mathcal{H}^{3}\left[G_{f}, U_{T}(1)\right] \rightarrow H_{f}^{3}\left[G_{f}, U_{T}(1)\right] \rightarrow H^{1}\left(G_{b}, \mathbb{Z}_{2}\right) \rightarrow 0$ \\
3 & $0 \rightarrow H_{\text {rigid }}^{4}\left[G_{b}, U_{T}(1)\right] \rightarrow \mathcal{H}^{4}\left[G_{f}, U_{T}(1)\right] \rightarrow B H^{3}\left(G_{b}, \mathbb{Z}_{2}\right) \rightarrow 0$ \\
& $0 \rightarrow \mathcal{H}^{4}\left[G_{f}, U_{T}(1)\right] \rightarrow H_{f}^{4}\left[G_{f}, U_{T}(1)\right] \rightarrow \tilde{B} H^{2}\left(G_{b}, \mathbb{Z}_{2}\right) \rightarrow 0$ \\
\hline \hline
\end{tabular}

in Sec. IV B. $B H^{3}\left(G_{b}, \mathbb{Z}_{2}\right)$, the obstruction-free subgroup of $H^{3}\left(G_{b}, \mathbb{Z}_{2}\right)$, is formed by elements $n_{3} \in H^{3}\left(G_{b}, \mathbb{Z}_{2}\right)$ that satisfy $S q^{2}\left(n_{3}\right)=0$ in $H^{4}\left(G_{b}, U_{T}(1)\right)$. We note that $B H^{3}\left(G_{b}, \mathbb{Z}_{2}\right)$ and $H_{\text {rigid }}^{4}\left(G_{b}, U_{T}(1)\right) \equiv H^{4}\left(G_{b}, \mathbb{Z}_{2}\right) / \Gamma$ were derived in the special group supercohomology classification. Recall that $H^{4}\left(G_{b}, U_{T}(1)\right)$ is the well-known classification of bosonic SPT phases and $\Gamma$ is a normal subgroup of $H^{4}\left(G_{b}, U_{T}(1)\right)$ generated by $S q^{2}\left(n_{2}\right)$, where $n_{2} \in$ $H^{2}\left(G_{b}, \mathbb{Z}_{2}\right)$ and $S q^{2}\left(n_{2}\right)$ are viewed as elements of $H^{4}\left[G_{b}, U_{T}(1)\right]$. Physically, $\Gamma$ describes those trivialized bosonic SPT phases when embedded into interacting fermion systems.

Together with several previous works $[42,50]$, we conjecture that up to spacial dimension $d_{\mathrm{sp}}=3$, FSPT with symmetry $G_{f}=G_{b} \times \mathbb{Z}_{2}^{f}$ can be classified by the general group supercohomology class $H_{f}^{d_{\text {sp }}+1}\left[G_{f}, U_{T}(1)\right]$ defined by the exact sequences summarized in Table I. We note that for spacial dimension $d_{\mathrm{sp}}>1$, general group supercohomology theory is defined by two short exact sequences. The first short exact sequence can be understood as decoration of complex fermions onto the intersection points of the $G_{b^{-}}$ symmetry domain walls, which was first derived by special group supercohomology theory. The second exact sequence can be understood as decoration of Kitaev's Majorana chains onto the intersection lines of $G_{b}$-symmetry domain walls, and our construction gives rise to a general scheme to compute $\tilde{B} H^{d_{\mathrm{sp}}-1}\left(G_{b}, \mathbb{Z}_{2}\right)$ [the obstruction-free subgroup of $\left.H^{d_{\mathrm{sp}}-1}\left(G_{b}, \mathbb{Z}_{2}\right)\right]$ in arbitrary dimensions. As an application, we also illustrate the classification results of FSPT phases for some simple symmetry group $G_{b}$ in all physical dimensions in Table II.

Finally, regarding the completeness of general group supercohomology classification for 3D FSPT phases, we present some physical arguments. Although the decoration of complex fermions on the intersection points of $G_{b^{-}}$ symmetry domain walls and the decoration of Majorana chains on $G_{b}$-symmetry domain walls give rise to a complete classification of 2D FSPT phases, this does not necessarily imply that this is also true in 3D. In fact, it has been pointed out [8] that the decoration of invertible TQFT on the $G_{b}$-symmetry domain walls may also give rise to new SPT states. For bosonic SPT states, decoration of the so-called $E_{8}$ state on the $G_{b}$-symmetry domain walls indeed produces the efmf SPT state beyond group cohomology classification. It has also been pointed out that $H^{1}\left(G_{b}, \mathbb{Z}\right)$ classifies these additional bosonic SPT states. As $H^{1}\left(G_{b}, \mathbb{Z}\right)$ is trivial for the unitary symmetry group $G_{b}$ and $H^{1}\left(\mathbb{Z}_{2}^{T}, \mathbb{Z}\right)=\mathbb{Z}_{2}$ for the antiunitary timereversal symmetry, we understand why the ef $m f$ state is the only nontrivial root state of bosonic SPT states beyond group cohomology classification with time-reversal symmetry. For interacting fermion systems, in principle, we can decorate a $p+i p$ state (the root state of $2 \mathrm{D}$ fermionic invertible TQFT) onto the $G_{b}$-symmetry domain walls. However, as $H^{1}\left(G_{b}, \mathbb{Z}\right)$ is trivial for the unitary symmetry group $G_{b}$, there are no new FSPT states with unitary symmetry group $G_{b}$. For time-reversal symmetry, it is possible to generate new FSPT states in this way, and we discuss this possibility in our future work.

TABLE II. Classification of FSPT phases with a total symmetry $G_{f}=G_{b} \times \mathbb{Z}_{2}^{f}$ in $d_{\mathrm{sp}}$ spatial dimensions constructed using general group supercohomoloy for some simple symmetries (represented by the bosonic symmetry groups $G_{b}$ ). Here, $\mathbb{Z}_{1}$ means that our construction only gives rise to the trivial phase. $\mathbb{Z}_{n}$ means that the constructed nontrivial SPT phases plus the trivial phase are labeled by the elements in $\mathbb{Z}_{n}$.

\begin{tabular}{|c|c|c|c|c|}
\hline$\underline{G_{b} \backslash d_{s p}}$ & 0 & 1 & 2 & 3 \\
\hline $\mathbb{Z}_{2}$ & $\mathbb{Z}_{2}^{2}$ & $\mathbb{Z}_{2}$ & $\mathbb{Z}_{8}$ & $\mathbb{Z}_{1}$ \\
\hline $\mathbb{Z}_{2 k+1}$ & $\mathbb{Z}_{4 k+2}$ & $\mathbb{Z}_{1}$ & $\mathbb{Z}_{2 k+1}$ & $\mathbb{Z}_{1}$ \\
\hline $\mathbb{Z}_{2 k}$ & $\mathbb{Z}_{2 k} \times \mathbb{Z}_{2}$ & $\mathbb{Z}_{2}$ & $\begin{cases}\mathbb{Z}_{4 k} \times \mathbb{Z}_{2}, & k \text { even } \\
\mathbb{Z}_{8 k}, & k \text { odd }\end{cases}$ & $\mathbb{Z}_{1}$ \\
\hline $\mathbb{Z}_{2} \times \mathbb{Z}_{2}$ & $\left(\mathbb{Z}_{2}\right)^{3}$ & $\left(\mathbb{Z}_{2}\right)^{3}$ & $\left(\mathbb{Z}_{8}\right)^{2} \times \mathbb{Z}_{4}$ & $\left(\mathbb{Z}_{2}\right)^{2}$ \\
\hline $\mathbb{Z}_{2} \times \mathbb{Z}_{4}$ & $\mathbb{Z}_{4} \times\left(\mathbb{Z}_{2}\right)^{2}$ & $\left(\mathbb{Z}_{2}\right)^{3}$ & $\left(\mathbb{Z}_{8}\right)^{2} \times\left(\mathbb{Z}_{2}\right)^{3}$ & $\mathbb{Z}_{4} \times \mathbb{Z}_{2}$ \\
\hline $\mathbb{Z}_{4} \times \mathbb{Z}_{4}$ & $\left(\mathbb{Z}_{4}\right)^{2} \times \mathbb{Z}_{2}$ & $\left(\mathbb{Z}_{2}\right)^{2} \times \mathbb{Z}_{4}$ & $\left(\mathbb{Z}_{8}\right)^{2} \times \mathbb{Z}_{4} \times\left(\mathbb{Z}_{2}\right)^{3}$ & $\left(\mathbb{Z}_{4}\right)^{2} \times \mathbb{Z}_{2}$ \\
\hline $\mathbb{Z}_{2} \times \mathbb{Z}_{8}$ & $\mathbb{Z}_{8} \times\left(\mathbb{Z}_{2}\right)^{2}$ & $\left(\mathbb{Z}_{2}\right)^{3}$ & $\mathbb{Z}_{16} \times \mathbb{Z}_{8} \times\left(\mathbb{Z}_{2}\right)^{3}$ & $\mathbb{Z}_{8} \times \mathbb{Z}_{2}$ \\
\hline
\end{tabular}




\section{Organization of the paper}

The remainder of this paper is organized as follows. We begin with the definition of Hilbert space and the basic structure of fixed-point wave functions for FSPT states with total symmetry $G_{f}=G_{b} \times \mathbb{Z}_{2}^{f}$ in $1 \mathrm{D}, 2 \mathrm{D}$, and 3D in Sec. II. In Sec. III A, we give a brief review of discrete spin structures and Kasteleyn orientations in 2D. In Sec. III B, we derive the fixed-point conditions for FSLU transformations under wave function renormalization and rederive the classifications of 2D FSPT phases. In Sec. IV A, we discuss how to generalize the discrete spin structure and local Kasteleyn orientation in 3D. In Sec. IV B, we use the concept of equivalence class of FSLU transformations and wave function renormalization to obtain the construction and classification of 3D FSPT phases. Finally, we offer conclusions and discussions for possible future directions in Sec. V.

Readers less interested in the detailed mathematical construction of (local) Kasteleyn orientations are invited to skip some part of Secs. III A and IV A and read directly Secs. III B and IV B of constructing FSPT states. The only prerequisites are some terminology conventions and the conclusion that we can construct (local) Kasteleyn orientations systematically and rigorously on the resolved dual lattice of arbitrary triangulations of spin manifolds in arbitrary dimensions.

\section{FIXED-POINT WAVE FUNCTIONS OF FSPT PHASES}

\section{A. Constructing fixed-point wave function and classification for FSPT phases in 1D}

As a warm-up, let us begin with fixed-point wave function in 1D and use FSLU transformation to rederive the well-known classification result of 1D FSPT phases. The building block of bosonic and fermionic degrees of freedom in the 1D FSPT model is shown in Fig. 1. Similar to the bosonic SPT phase, every (locally ordered) vertex $i$ of the 1D lattice has bosonic degrees of freedom labeled by a group element $g_{i} \in G_{b}$. (Recall that the FSPT phases have a total symmetry $G_{f}=G_{b} \times \mathbb{Z}_{2}^{f}$.) A spinless complex fermion $c_{(i j)}$ is at the center of each link $\langle i j\rangle$ (see the blue ball in Fig. 1), and the fermion occupation number $n_{1}\left(g_{i}, g_{j}\right)$ is either 0 or 1 . Let $|0\rangle$ be the ground state of no fermions on any of the links; then, a generating set of the Fock space is given by $\prod_{(i j) \in l} c_{(i j)}^{\dagger}|0\rangle$, where $l \subset L$ is a

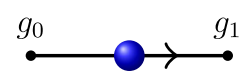

FIG. 1. Bosonic and fermionic degrees of freedom for 1D fixedpoint FSPT states on a link. The black dots are bosonic degrees of freedom labeled by $g_{i} \in G$ on sites. The blue ball represents the complex fermion $c_{(i j)}$ at the center of the link $\langle i j\rangle$. The arrow represents the local order of two sites. subset of all links $L$, including the empty set. Thus, the full local Hilbert space for our 1D model on a fixed lattice $\mathcal{T}$ (triangulation of 1D spacial manifold) is

$$
L_{\mathcal{T}}^{1 \mathrm{D}}=\bigoplus_{l \subset L}\left(\prod_{(i j) \in l} c_{(i j)}^{\dagger}|0\rangle \otimes \prod_{v \in V(\mathcal{T})} \mathbb{C}^{\left|G_{b}\right|}\right) .
$$

Here, $\left|G_{b}\right|$ is the order of the bosonic symmetry group $G_{b}$. As a vector space, the fermionic Hilbert space on the links is the same as the tensor product $\bigotimes_{L(\mathcal{T})} \mathbb{C}^{2}$; however, the Fock space structure means that a local Hamiltonian for a fermion system is nonlocal when regarded as one for a boson system. We note that the structure of total bosonic and fermionic Hilbert space on arbitrary triangulations is the same as the 1D case of Ref. [42], although the latter is considering the spacetime picture.

Our 1D fixed-point state is a superposition of those basis states with all possible triangulations $\mathcal{T}$ :

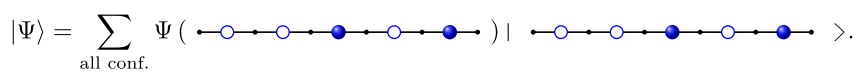

In the following, we derive the rules of wave function renormalization generated by FSLU transformations for the above wave function. We obtain the conditions for a fixedpoint wave function and show how to construct all FSPT states with total symmetry $G_{f}=G_{b} \times \mathbb{Z}_{2}^{f}$ in $1 \mathrm{D}$.

\section{Fermionic symmetric local unitary transformation}

To obtain a fixed-point wave function for Eq. (5), we need to understand the changes of the wave function under renormalization. In $1 \mathrm{D}$, renormalization can be understood as removing some bosonic or fermionic degrees of freedom by reducing the number of vertices. The basic renormalization process is known as (2-1) Pachner move of triangulation of 1D manifold. Since we have a bosonic degree of freedom at each vertex and a fermionic degree of freedom at each link, the (2-1) move effectively reduces the Hilbert space of one bosonic mode and one fermionic mode.

To be more precise, the (2-1) move is an FSLU transformation between the fermionic Fock spaces on two different triangulations:

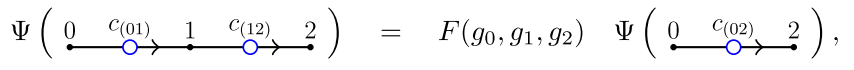

where the $F$ operator is defined as

$$
\begin{aligned}
F\left(g_{0}, g_{1}, g_{2}\right)= & \frac{1}{\left|G_{b}\right|^{1 / 2}} \nu_{2}\left(g_{0}, g_{1}, g_{2}\right) c_{(12)}^{\dagger n_{1}\left(g_{1}, g_{2}\right)} \\
& \times c_{(01)}^{\dagger n_{1}\left(g_{0}, g_{1}\right)} c_{(02)}^{n_{1}\left(g_{0}, g_{2}\right)} .
\end{aligned}
$$


We note that the $\left|G_{b}\right|$ is the order of the group $G_{b}$, and we introduce the normalization factor $1 /\left|G_{b}\right|^{1 / 2}$ in the above expression due to the change of vertex number. Here, $\nu_{2}\left(g_{0}, g_{1}, g_{2}\right)$ is a $U_{T}(1)$-valued function with variables $g_{i} \in G_{b}$ and $c_{(i j)}^{\dagger}$ is the creation operator for $c$ fermions at link $\langle i j\rangle . \quad n_{1}\left(g_{i}, g_{j}\right) \in\{0,1\}$ is a $\mathbb{Z}_{2}$-valued function indicating whether there is a $c$ fermion at link $\langle i j\rangle$ or not. Since we are constructing a symmetric state, both $\nu_{2}$ and $n_{1}$ should be symmetric under the action of $G_{b}$ [we note that $\nu_{2}\left(g g_{0}, g g_{1}, g g_{2}\right)=\nu_{2}^{*}\left(g_{0}, g_{1}, g_{2}\right)$ if $g$ is antiunitary]. So they are $U_{T}(1)$-valued 2-cochain and $\mathbb{Z}_{2}$-valued 1cochain, respectively. Because the renormalization process (2-1) move should preserve the fermion parity, we have $d n_{1}\left(g_{0}, g_{1}, g_{2}\right)=n_{1}\left(g_{1}, g_{2}\right)+n_{1}\left(g_{0}, g_{2}\right)+n_{1}\left(g_{0}, g_{1}\right)=0$ $(\bmod 2)$. Therefore, $n_{1}$ is in fact a $\mathbb{Z}_{2}$-valued 1-cocycle.

\section{Consistent equations and equivalence classes}

Since we are constructing a fixed-point wave function, Eq. (5) should be invariant under renormalization. For instance, we can use two different sequences of $F$ moves Eq. (6) to connect a fixed initial state and a fixed final state. Different approaches should give rise to the same wave function. These constraints give us the consistent equations for $\nu_{2}$.

The simplest example is the following two paths between two fixed states:

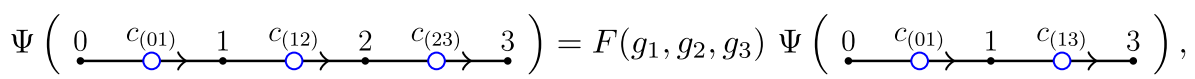

$$
\begin{aligned}
& =F\left(g_{1}, g_{2}, g_{3}\right) F\left(g_{0}, g_{1}, g_{3}\right) \Psi\left(\stackrel{\left(\begin{array}{lll}
0 & c_{(03)} & \longrightarrow
\end{array}\right.}{\longrightarrow}\right) \text {, }
\end{aligned}
$$

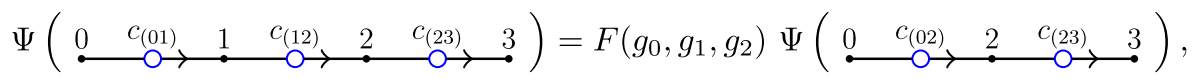

$$
\begin{aligned}
& =F\left(g_{0}, g_{1}, g_{2}\right) F\left(g_{0}, g_{2}, g_{3}\right) \Psi\left(\stackrel{\left(\begin{array}{lll}
0 & c_{(03)} & 3
\end{array}\right.}{\longrightarrow}\right) \text {. }
\end{aligned}
$$

The constraint is that the product of $F$ moves for the above two processes equals to each other:

$$
F\left(g_{0}, g_{1}, g_{3}\right) F\left(g_{1}, g_{2}, g_{3}\right)=F\left(g_{0}, g_{2}, g_{3}\right) F\left(g_{0}, g_{1}, g_{2}\right) \text {. }
$$

Substituting the expression of $F$ move Eq. (7) into this equation and using the fact $d n_{1}=0(\bmod 2)$, we find that the above equation for fermionic operators is equivalent to a purely bosonic one without any fermion sign:

$$
d \nu_{2}\left(g_{0}, g_{1}, g_{2}, g_{3}\right)=\frac{\nu_{2}\left(g_{1}, g_{2}, g_{3}\right) \nu_{2}\left(g_{0}, g_{1}, g_{3}\right)}{\nu_{2}\left(g_{0}, g_{2}, g_{3}\right) \nu_{2}\left(g_{0}, g_{1}, g_{2}\right)}=1 \text {. }
$$

That means $\nu_{2}$ should be a $U_{T}(1)$-valued 2-cocycle, provided that the wave function Eq. (5) is a fix-point wave function. These $\nu_{2}$ data are the same as the construction of bosonic SPT states.

Using an FSLU transformation, we can redefine the basis state $\left|\left\{g_{l}\right\}\right\rangle$ as

$$
\begin{aligned}
\left|\left\{g_{l}\right\}\right\rangle^{\prime}= & U_{\mu_{1}, m_{0}}\left|\left\{g_{l}\right\}\right\rangle \\
= & \prod_{\langle i j\rangle} \mu_{1}\left(g_{i}, g_{j}\right) \prod_{\langle i\rangle}\left[f_{i A}^{m_{0}\left(g_{i}\right)} f_{i B}^{m_{0}\left(g_{i}\right)}\right] \\
& \times \prod_{\langle i j\rangle}\left[f_{j A}^{\dagger m_{0}\left(g_{j}\right)} f_{i B}^{\dagger m_{0}\left(g_{i}\right)}\right]\left|\left\{g_{l}\right\}\right\rangle,
\end{aligned}
$$

where we first create two complex fermions $f_{j A}$ and $f_{i B}$ near the two ends of the link $\langle i j\rangle(i<j)$, and then annihilate the two fermions $f_{i A}$ and $f_{i B}$ near the vertex $i$ when gluing the two links sharing vertex $i$. To preserve the fermion parity and be symmetric, $m_{0}$ should be a 0 -cocycle (with $\mathbb{Z}_{2}$ coefficient): $m_{0}\left(g g_{i}\right)=m_{0}\left(g_{i}\right)$ and $d m_{0}\left(g_{i}, g_{j}\right)=$ $m_{0}\left(g_{j}\right)+m_{0}\left(g_{i}\right)=0 . \mu_{1}\left(g_{i}, g_{j}\right)$ is a phase factor associated with link $\langle i j\rangle$. In this new basis, the fermionic $F$ move is $F^{\prime}=U_{\mu_{1}, m_{0}} F U_{\mu_{1}, m_{0}}^{\dagger}$. After eliminating all $f$ fermions (all the fermion signs are canceled), one finds that the phase factor in Eq. (7) becomes

$\nu_{2}^{\prime}\left(g_{0}, g_{1}, g_{2}\right)=\nu_{2}\left(g_{0}, g_{1}, g_{2}\right) \frac{\mu_{1}\left(g_{1}, g_{2}\right) \mu_{1}\left(g_{0}, g_{1}\right)}{\mu_{1}\left(g_{0}, g_{2}\right)}$.

Since our gapped phases are defined by FSLU transformations, $\nu_{2}^{\prime}$ and $\nu_{2}$ belong to the same phase. In general, the elements $\nu_{2}$ in the same group cohomology class in $H^{2}\left(G_{b}, U_{T}(1)\right)$ correspond to the same 1D FSPT phase. This is consistent with the general result obtained from the path-integral formalism in Ref. [42] that $\nu_{d+1}$ can be gauge transformed to

$$
\nu_{d+1}^{\prime}=\nu_{d+1} d \mu_{d}(-1)^{S q^{2}\left(m_{d-1}\right)},
$$

for $S q^{2}\left(m_{0}\right)$ is trivial in the 1D FSPT case. 
In summary, 1D FSPT is characterized by $n_{1} \in$ $H^{1}\left(G_{b}, \mathbb{Z}_{2}\right)$ and $\nu_{2} \in H^{2}\left(G_{b}, U_{T}(1)\right)$. This is consistent with the previous result [42].

\section{B. Constructing fixed-point wave function for FSPT phases in 2D and 3D}

The fixed-point wave functions for FSPT phases in 2D and $3 \mathrm{D}$ are much more complicated. We describe all the details and explain the corresponding physical meanings below.

Similar to the wave function renormalization scheme for 2D bosonic SET phases, we consider the quantum state defined on an arbitrary triangulation for 2D and 3D FSPT phases. The triangulation admits a branching structure that can be labeled by a set of local arrows on all links (edges) with no oriented loop for any triangle. Mathematically, the branching structure can be regarded as a discrete version of a $\operatorname{spin}^{\mathrm{c}}$ structure and can be consistently defined on arbitrary triangulations of 2D and 3D orientable manifolds.

We begin with the construction of a fixed-point wave function in 2D; then, the generalization to 3D becomes straightforward. As any 2D FSPT state can be naturally mapped to a 2D bosonic SET state by gauging the fermion parity symmetry, our construction for fixed-point wave functions is greatly inspired by such connections. In particular, an FSPT state with total symmetry $G_{f}=G_{b} \times$ $\mathbb{Z}_{2}^{f}$ can be mapped to a $G_{b}$ symmetry-enriched toric code model. As a simple example, fixed-point wave functions and commuting projector parent Hamiltonians on an arbitrary trivalent graph (due to triangulation) with $G_{b}=$ $\mathbb{Z}_{2}$ were constructed in Ref. [61]. It has also been shown that all of these SET states can be obtained by gauging fermion parity from FSPT states with total symmetry $G_{f}=\mathbb{Z}_{2} \times \mathbb{Z}_{2}^{f}$.

The building block of bosonic and fermionic degrees of freedom in our 2D FSPT model is shown in Fig. 2. Exactly as in the bosonic SET phase, every vertex $i$ of the space triangulation has bosonic degrees of freedom labeled by a

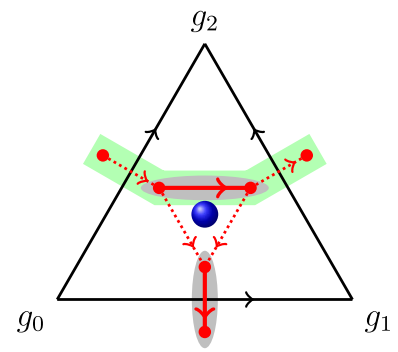

FIG. 2. Fermionic degrees of freedom in a triangle. The red dots represent Majorana fermions at the two sides of each link. The blue ball represents the complex fermion of the special group supercohomology model at the center of the triangle. The green strip is the decorated Kitaev's Majorana chain onto the dual lattice $\mathcal{P}$. group element $g_{i} \in G_{b}$. (Recall that the FSPT phases have a total symmetry $G_{f}=G_{b} \times \mathbb{Z}_{2}^{f}$.) A spinless complex fermion $c$ is at the center of each triangle or face (see the blue ball in Fig. 2), and the fermion occupation number is either 0 or 1 . Let $|0\rangle$ be the ground state of no fermions on any of the triangles; then, a generating set of the Fock space is given by $\prod_{(i j k) \in f} c_{(i j k)}^{\dagger}|0\rangle$, where $f \subset F$ is a subset of all triangles $F$, including the empty set. In addition, each link has two Majorana fermions on its two sides, an arrangement that is equivalent to spinless complex fermion $a$. Similar to the $c$ fermion on each triangle, let $|\tilde{0}\rangle$ be the ground state of no fermions on any of the links; then, a generating set of the Fock space is given by $\prod_{(i j) \in l} a_{(i j)}^{\dagger}|\tilde{0}\rangle$, where $l \subset L$ is a subset of all links $L$, including the empty set. Thus, the full local Hilbert space for our 2D model on a fixed triangulation $\mathcal{T}$ is

$\left.L_{\mathcal{T}}^{2 \mathrm{D}}=\bigoplus_{f \subset F l \subset L} \bigoplus_{(i j k) \in f} c_{(i j k)}^{\dagger}|0\rangle \otimes \prod_{(i j) \in l} a_{(i j)}^{\dagger}|\tilde{0}\rangle \otimes \prod_{v \in V(\mathcal{T})} \mathbb{C}^{\left|G_{b}\right|}\right)$.

Here, $\left|G_{b}\right|$ is the order of the bosonic symmetry group $G_{b}$. As a vector space, the fermionic Hilbert space on the triangles and links is the same as the tensor product $\bigotimes_{F(\mathcal{T})} \mathbb{C}^{2} \otimes \bigotimes_{L(\mathcal{T})} \mathbb{C}^{2}$; however, the Fock space structure means that a local Hamiltonian for a fermion system is nonlocal when regarded as one for a boson system. We note that the structure of total fermionic Hilbert space on arbitrary triangulations is slightly more general than that given in Ref. [63]; this allows us to construct very general FSET states in 2D. However, the construction of general FSET states is beyond the scope of this paper.

As mentioned above, for FSPT states, the support space of FSLU transformations must be one dimensional such that it can adiabatically connect to a product state in the absence of global symmetry. Therefore, the fermionic states of $c$ and $a$ fermions on the triangles and edges are completely fixed by the configuration of group elements $\left\{g_{i}\right\}$ on the vertices. In particular, the equivalence classes of complex fermion occupation number of $c$ fermions are uniquely determined by the elements in $B H^{2}\left(G_{b}, \mathbb{Z}_{2}\right)$ [the obstruction-free subgroup of $H^{2}\left(G_{b}, \mathbb{Z}_{2}\right)$ ], which was first proposed by the special group supercohomology construction of FSPT phases. Essentially, the complex fermion $c$ can be regarded as a decoration on the intersection points of $G_{b}$-symmetry domain walls. References $[50,67,68]$ pointed out that a Majorana chain can be decorated onto the $G_{b^{-}}$ symmetry domain walls to generate a complete set of FSPT states in 2D. This layer of decoration is uniquely determined by the elements in $H^{1}\left(G_{b}, \mathbb{Z}_{2}\right)$. The Majorana fermions must be paired (see the gray ellipse in Fig. 2) to form Kitaev's Majorana chains on the $G_{b}$ symmetry domain walls (see the green strip in Fig. 2). This requires a 
discrete spin structure-the Kasteleyn orientations on the dual trivalent lattice (with proper resolution for the lattice sites, as seen in Fig. 2) - such that the total fermion parity of the $a$ fermion is always even on any closed loop. We review all of the details in Sec. III A. An example of triangulation of the torus and decoration of Kitaev's Majorana chains is given in Fig. 4. The full details are discussed in Sec. III B.

Thus, our 2D fixed-point state is a superposition of those basis states with all possible triangulations $\mathcal{T}$ :

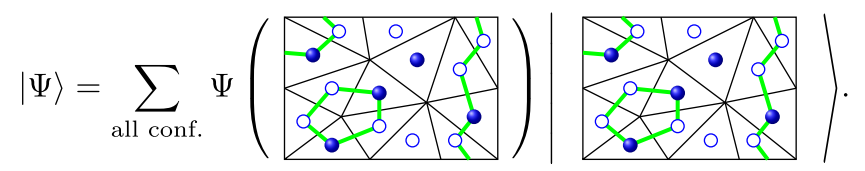

In Sec. III B, we derive the rules of wave function renormalization generated by FSLU transformations. We also obtain the conditions for fixed-point wave functions and show how to construct all FSPT states with total symmetry $G_{f}=G_{b} \times \mathbb{Z}_{2}^{f}$ on arbitrary triangulations in $2 \mathrm{D}$.

In the following, we generalize all of the above constructions to 3D. The building block of bosonic and fermionic degrees of freedom is shown in Fig. 3. Again, every vertex $i$ of the 3D space triangulation has a bosonic degree of freedom labeled by a group element $g_{i} \in G_{b}$. However, the spinless complex fermion $c$ introduced by special group supercohomology theory now resides on each tetrahedron (see the blue ball in Fig. 3). In addition, each triangle of the space tetrahedron has two Majorana fermions on its two sides, which is again equivalent to a spinless complex fermion $a$. Similar to the 2D case, let $|0\rangle$ and $|\tilde{0}\rangle$ be the ground states of no fermions on any tetrahedron and triangle; then, a generating set of the Fock space is given by $\prod_{(i j k l) \in t} c_{(i j k l)}^{\dagger}|0\rangle \otimes \prod_{(i j k) \in f} a_{(i j k)}^{\dagger}|\tilde{0}\rangle$, where $t \subset T$ is a subset of all tetrahedra $T$, including the empty set, and $f \subset F$ is

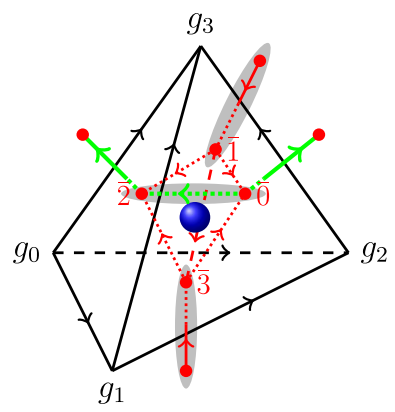

FIG. 3. Fermionic degrees of freedom in a tetrahedron. The red dots represent Majorana fermions on the two sides of each triangle. The blue ball represents the complex fermion of the (special) group supercohomology model at the center of the tetrahedron. The green line is the decorated Kitaev's Majorana chain on the dual lattice $\mathcal{P}$. a subset of all triangles $F$, including the empty set. Thus, the full local Hilbert space of our 3D model on a fixed triangulation $\mathcal{T}$ is

$$
\begin{aligned}
L_{\mathcal{T}}^{3 \mathrm{D}} & =\bigoplus_{t \subset T} \bigoplus_{f \subset F}\left(\prod_{(i j k l) \in t} c_{(i j k l)}^{\dagger}|0\rangle \otimes \prod_{(i j k) \in f} a_{(i j k)}^{\dagger}|\tilde{0}\rangle\right. \\
& \left.\otimes \prod_{v \in V(\mathcal{T})} \mathbb{C}^{\left|G_{b}\right|}\right) .
\end{aligned}
$$

Similar to the 2D case, the fermionic states of $c$ and $a$ fermions on the tetrahedra and triangles are also completely fixed by the configuration of group elements $\left\{g_{i}\right\}$ on the vertices. The equivalence classes of complex fermion occupation number of the $c$ fermion are uniquely determined by the elements in $B H^{3}\left(G_{b}, \mathbb{Z}_{2}\right)$ [the obstructionfree subgroup of $H^{3}\left(G_{b}, \mathbb{Z}_{2}\right)$ ], which was also first proposed by the special group supercohomology construction of FSPT states. It is not a surprise that in 3D the complex fermion $c$ can also be regarded as a decoration (subjected to obstructions) onto the intersection points of $G_{b}$-symmetry domain walls. The most interesting new feature here is that a Majorana chain can also be decorated onto the intersection lines of $G_{b}$-symmetry domain walls, and such a construction generates a new set of FSPT states in 3D. As expected, this layer of decoration also requires a discrete spin structure on the dual trivalent lattice (with a proper resolution for the lattice sites as well, as seen in Fig. 3), and the Majorana fermions must also be paired to form Kitaev's Majorana chains (see the green line in Fig. 3). However, such decorations are subjected to a fundamental obstruction on $H^{4}\left(G_{b}, \mathbb{Z}_{2}\right)$ due to fermion parity conservation. We discuss all of the details in Sec. IVA. Furthermore, the fixed-point condition of wave function renormalization gives rise to a secondary obstruction on $H^{5}\left(G_{b}, U_{T}(1)\right)$, which is explored in full in Sec. IV B.

Finally, our 3D fixed-point state is a superposition of those basis states with all possible triangulations $\mathcal{T}$ :

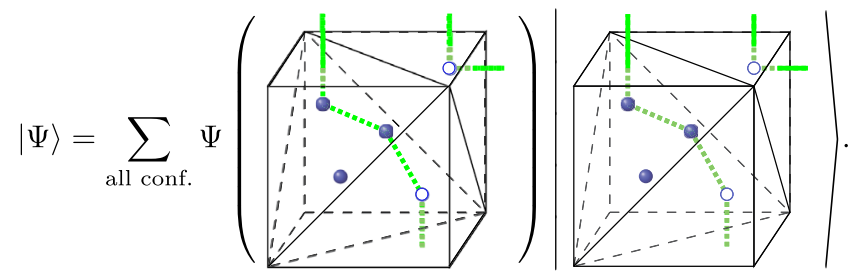

In Sec. IV B, we derive the rules of wave function renormalization generated by FSLU transformations. We also obtain the conditions for fixed-point wave function and show how to construct all FSPT states with total symmetry $G_{f}=G_{b} \times \mathbb{Z}_{2}^{f}$ on arbitrary triangulations in 3D. 


\section{CONSTRUCTIONS AND CLASSIFICATIONS FOR FSPT STATES IN 2D}

Reference [67] pointed out that discrete spin structures and Kasteleyn orientation played an essential role in constructing FSPT phases decorated with Kitaev's Majorana chains on $G_{b}$-symmetry domain walls. In this section, we give a brief review of the essential idea and generalize the construction to arbitrary triangulations in 2D (see Fig. 4). In particular, we use Poincaré dual to show how to implement discrete spin structures and Kasteleyn orientation for an arbitrary triangulation with a branching structure in 2D. Essentially, the Poincaré dual enables us to define discrete spin structures in arbitrary dimensions and gives rise to the notion of local Kasteleyn orientation, which serves as the key step toward decorating Kitaev's Majorana chains onto the intersection lines of $G_{b^{-}}$ symmetry domain walls in $3 \mathrm{D}$.

In the following, we start by defining spin structures in terms of the second Stiefel-Whitney class. Then, we clarify the relation between the discrete spin structures and the Kasteleyn orientation in 2D. Finally, we make use of the novel concept of equivalence classes of FSLU transformations (with a one-dimensional support space) to obtain the full classification of FSPT states with total symmetry $G_{f}=G_{b} \times \mathbb{Z}_{2}^{f}$ in $2 \mathrm{D}$.

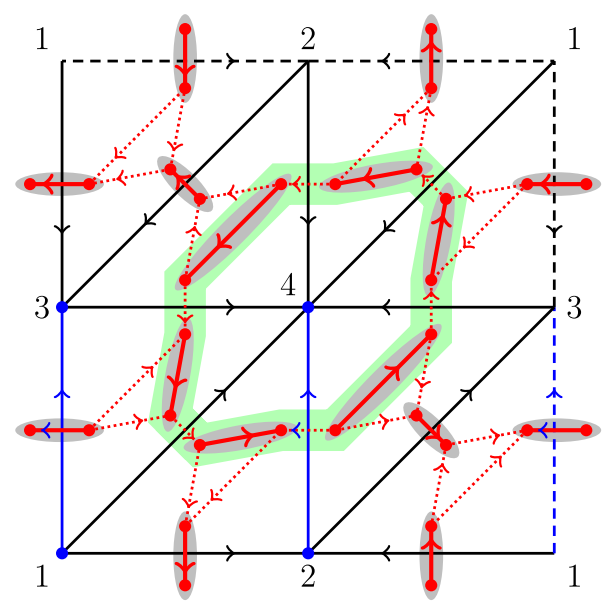

FIG. 4. Example of triangulation $\mathcal{T}$ of torus and Kitaev chain decoration. All vertices $\langle 1\rangle,\langle 2\rangle,\langle 3\rangle$, and $\langle 4\rangle$ (blue dots) are singular vertices; i.e., $w_{0}=\langle 1\rangle+\langle 2\rangle+\langle 3\rangle+\langle 4\rangle$. We choose link $\langle 13\rangle$ and $\langle 24\rangle$ (blue lines) to be singular lines; i.e., $w_{0}=\partial(\langle 13\rangle+\langle 24\rangle)$. The direction of the red links dual to $\langle 13\rangle$ and $\langle 24\rangle$ are changed. Vertices $i=1,2,3$, and 4 of $\mathcal{T}$ are labeled by group elements $g_{i} \in G$. Majorana fermions (red dots) reside on the vertices of the resolved dual lattice $\tilde{\mathcal{P}}$ (solid and dashed red links). The solid red links and gray ellipses indicate that the two Majorana fermions at their two ends are paired with respect to the link direction. The green strip is the $\mathbb{Z}_{2}$ domain wall of the "spin" configuration $\left\{g_{i}\right\}$ and is decorated by a Kitaev's Majorana chain (Majorana fermions along the domain wall are paired differently from the "vacuum").

\section{A. Discrete spin structure and Kasteleyn orientations}

In this section, we construct the zeroth Stiefel-Whitney homology class on arbitrary 2D triangulation lattice and relate it to the Kasteleyn orientations on the resolved dual lattice. The general procedures of constructing Kasteleyn orientations are summarized as follows.

(1) Given a (black) triangulation lattice $\mathcal{T}$ with branching structure for a 2D spin manifold,

(2) Construct the (red) resolved dual lattice $\tilde{\mathcal{P}}$ and (red) link orientations using convention Fig. 6. At this stage, some of the vertices in $\mathcal{T}$ are non-Kasteleyn oriented.

(3) Find the expression of $w_{0}$ in Eq. (20) as a formal summation of singular vertices of $\mathcal{T}$ [i.e., non-Kasteleynoriented vertices in step (2)].

(4) Connect singular vertices in $\mathcal{T}$ by (blue) lines $S$ (i.e., $\partial S=w_{0}$ ).

(5) Using convention Fig. 7(b), reverse the orientations of (red) links dual to (blue) links belonging to $S$.

(6) Now all the vertices in $\mathcal{T}$ are Kasteleyn oriented.

After all the above steps, the resolved dual lattice $\tilde{\mathcal{P}}$ now has Kasteleyn orientations. In this way, any decorations of Kitaev's Majorana chains will have the same fermion parity. We use them to construct generic FSPT states in Sec. III B.

\section{Discrete Stiefel-Whitney homology class $w_{0}$}

It is well known that an oriented manifold $M$ (with dimension $n$ ) admits spin structures if and only if its second Stiefel-Whitney class $\left[w^{2}\right] \in H^{2}\left(M, \mathbb{Z}_{2}\right)$ vanishes. In the construction of the lattice models upon triangulation of $M$, we find it more convenient to use the $(n-2)$ th StiefelWhitney homology class $\left[w_{n-2}\right]$, which is the Poincaré dual of $\left[w^{2}\right]$.

In this section, we consider only the $2 \mathrm{D}$ case. For a spatial manifold $M(n=2)$ with triangulation $\mathcal{T}$, the Stiefel-Whitney homology class $\left[w_{0}\right]$ has a representative that is the summation of all vertices $v$ with some $(\bmod 2)$ coefficients as follows $[69,70]$ :

$$
w_{0}=\sum_{v \in \mathcal{T}} \mathcal{N}\{\sigma \mid v \subseteq \sigma \text { is regular }\} v .
$$

Here, $v \subseteq \sigma$ means that $v$ is a subsimplex of simplex $\sigma . v \subseteq \sigma$ is called regular if $v$ and $\sigma$ have one of the three relative positions shown in Fig. 5. $\mathcal{N}\{\sigma \mid v \subseteq \sigma$ is regular $\} \cdot v$ denotes the formal product of the $(\bmod 2)$ number of regular pairs $v \subseteq \sigma$ and the vertex $v$. We call vertex $v$ singular if $\mathcal{N}\{\sigma \mid v \subseteq \sigma$ is regular $\}$ is odd. In this language, $w_{0}$ in Eq. (19) is the formal summation of all singular vertices. $w_{0}$ is a vector (0th singular chain) in the vector space (of 0th singular chains) spanned by the formal bases of all vertices with $\mathbb{Z}_{2}$ coefficients.

On the other hand, it is known that all oriented 2D surfaces admit spin structures. Thus, the second StiefelWhitney class $\left[w^{2}\right]$ or the zeroth Stiefel-Whitney homology 

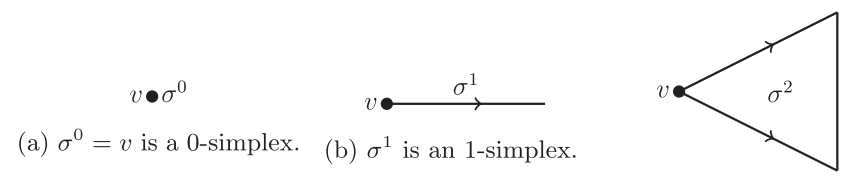

(c) $\sigma^{2}$ is a 2-simplex.

FIG. 5. Regular pair $v \subseteq \sigma^{i}(i=0,1,2)$ for vertex $v$.

class $\left[w_{0}\right]$ of any oriented surface is (co)homologically trivial. As a result, the collection of singular vertices $w_{0}$ in Eq. (19) can be viewed as a boundary $\partial S$ for some lines $S$ (we call them singular lines). The singular lines $S$ are colored blue in the following Figs. 7(b) and 8.

For a fixed collection of singular vertices (a fixed representative $w_{0}$ for $\left[w_{0}\right]$ ), different inequivalent choices of singular lines $S$ correspond to different spin structures that are isomorphic to $H^{1}\left(M, \mathbb{Z}_{2}\right)$ noncanonically. This can be seen as follows. We first choose arbitrary fixed $S_{0}$ such that $w_{0}=\partial S_{0}$. Then for any other choice $S$ with also $w_{0}=\partial S$, we can add $S_{0}$ and $S$ formally. The summation $S_{0}+S$ is a collection of closed loops on the manifold (recall that lines in $S_{0}$ and $S$ have the same end points). We can ask whether $S_{0}+S$ is in the trivial class of $H_{1}\left(M, \mathbb{Z}_{2}\right)$ or not. If it is, we say $S_{0}$ and $S$ are equivalent. In this way, with the fixed $S_{0}$, we have a one-to-one correspondence between equivalence classes of singular lines $S$ and $H_{1}\left(M, \mathbb{Z}_{2}\right)$. In other words, the set of equivalence classes of singular lines $S$ is an affine $H_{1}\left(M, \mathbb{Z}_{2}\right)$ space. This is also one of the most important properties of spin structures of a manifold. As a result, we have a one-to-one correspondence between $2^{2 g}$ equivalence classes of singular lines $S$ and $2^{2 g}$ spin structures of the manifold with genus $g$.

\section{Kasteleyn orientations and gauge transformations}

To decorate Kitaev chains onto domain walls of a 2D spin model, it is useful to determine the Kasteleyn orientation [64] for the edges of the lattice $[67,68]$. In this section, we relate the existence of discrete spin structures (the vanishing of $\left[w_{0}\right]$ ) of a triangulation $\mathcal{T}$ to the existence of Kasteleyn orientation of the resolved dual lattice. Then, in the next section, we use FSLU transformations $[28,63]$ to classify FSPT states and define exactly solvable models on arbitrary triangulations in 2D.

Our setup begins with a fixed triangulation $\mathcal{T}$ of the surface $M$. The first step is to construct a polyhedral decomposition $\mathcal{P}$ of $M$ that is a trivalent graph dual to $\mathcal{T}$. We add a spinless fermionic degree of freedom to every link of $\mathcal{T}$ and split it into two Majorana fermions on the two sides of this link for convenience. Equivalently, we can resolve the triangulation $\mathcal{T}$ by adding a new vertex to each triangle center and obtain a new triangulation $\tilde{\mathcal{T}}$. The Majorana fermions reside on the vertices of the resolved dual lattice $\tilde{\mathcal{P}}$, which is a trivalent graph dual to $\tilde{\mathcal{T}}$ (see Figs. 4 and 6 for this construction on a torus).

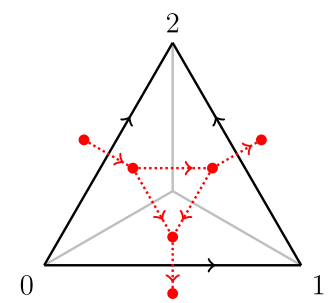

(a) Positive oriented triangle

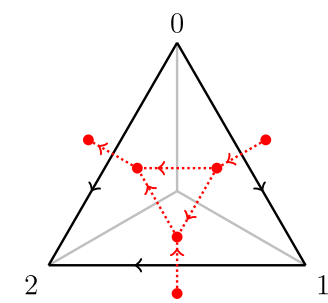

(b) Negative oriented triangle
FIG. 6. Triangulation $\mathcal{T}$ (black line), resolved triangulation $\tilde{\mathcal{T}}$ (black and gray line), and resolved dual lattice $\tilde{\mathcal{P}}$ (dashed red line). The resolved triangulation $\tilde{\mathcal{T}}$ was obtained from the original $\mathcal{T}$ by adding a new vertex to the center of each triangle. The links of $\mathcal{P}$ have orientations induced from the link orientations of $\mathcal{T}$ according to the conventions shown in Fig. 7(a). Red dots on the vertices of $\tilde{\mathcal{P}}$ represent Majorana fermions that split from the complex fermions on each link of $\mathcal{T}$ (see the discussion in Sec. III B).

The second step is adding directions to links in $\mathcal{T}$ and $\tilde{\mathcal{P}}$. We order all of the vertices in $\mathcal{T}$ and use the convention that all links are from vertices of smaller number to vertices of larger number. This is a branching structure of $\mathcal{T}$ such that there is no cycle for any triangle. The dual-link direction in $\mathcal{P}$ is obtained from $\mathcal{T}$ using the convention shown in Fig. 7(a). The directions of the new links in $\tilde{\mathcal{P}}$ are also obtained from triangulation $\mathcal{T}$ by using the conventions in Fig. 6.

The essential point of the aforementioned link orientation conventions can be explained as follows. When traveling along the smallest red loop in $\tilde{\mathcal{P}}$ around vertex $v \in \mathcal{T}$ counterclockwise, we encounter even numbers of red links (due to the resolvation) with the direction along or opposite to our direction (for example, the red loop inside the green strip around vertex 4 in Fig. 4). Using the conventions in Fig. 6, the red link direction is opposite the counterclockwise direction if and only if (1) the red link is dual to a black link in $\mathcal{T}$ such that $v$ is the initial point of this black link [this corresponds to the case in Fig. 5(b)] or (2) the red link is resolved to a new link inside a triangle in $\mathcal{T}$ such that $v$ is the first point of this triangle, i.e., the 0 point of triangle $\langle 012\rangle$ [this is the case in Fig. 5(c)]. If the

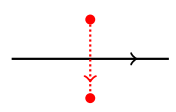

(a) Orientation convention for (red) (b) Orientation convention for (red) link dual to (black) link $l \notin S$. link dual to (blue) singular link $l \in S$.

FIG. 7. Conventions for the orientation of links in $\tilde{\mathcal{P}}$ (dotted red line) from branching structure of triangulation $\mathcal{T}$ (solid black line). Nonsingular (singular) black (blue) links $l \notin S(l \in S)$ induce orientation conventions for the dual link in $\tilde{\mathcal{P}}$. We introduce a spinless fermion on each (black or blue) link in $\mathcal{T}$ and split it into two Majorana fermions on the two sides of this link or vertices of $\tilde{\mathcal{P}}$ (red dots). 
total number of red links with opposite directions is odd, the vertex $v$ is considered Kasteleyn oriented. As the smallest loop in $\tilde{\mathcal{P}}$ around $v$ has an even number of red links, it does not matter whether we use counterclockwise or clockwise conventions. Under the above construction, we relate the zeroth Stiefel-Whitney homology class $w_{0}$ in Eq. (19) and the orientation of links in $\tilde{\mathcal{P}}$, i.e., $w_{0}$ is the summation of all non-Kasteleyn-oriented vertices:

$$
\begin{aligned}
w_{0}= & \sum_{v \in \mathcal{T}}\left(1+\mathcal{N}\left\{\sigma^{1} \mid v \subseteq \sigma^{1} \text { is regular }\right\}\right. \\
& \left.+\mathcal{N}\left\{\sigma^{2} \mid v \subseteq \sigma^{2} \text { is regular }\right\}\right) v \\
= & \sum_{v \in \mathcal{T}} v(v \text { is non-Kasteleyn oriented }) .
\end{aligned}
$$

As the zeroth Stiefel-Whitney homology class $\left[w_{0}\right]$ for any oriented surface is trivial, we have $w_{0}=\partial S$ for some singular line $S$. If we further reverse the direction of the links in $\tilde{\mathcal{P}}$, thus crossing the singular lines $S$ as shown in Fig. 7(b), then all of the vertices in $\mathcal{T}$ are Kasteleyn oriented, as this operation changes only the Kasteleyn property of the singular vertices in $w_{0}$ while preserving this property for all other vertices, including those in the interval of $S$. After completing the procedure above, we relate the vanishing of the zeroth Stiefel-Whitney homology class $\left[w_{0}\right]$ to the property of Kasteleyn orientation of the smallest loop around each vertex.

Note that the construction of link direction in $\tilde{\mathcal{P}}$ depends on the choice of the singular line $S$. On the one hand, the local shape of $S$ is not important as long as $\partial S$ is fixed. In fact, if we change the shape of $S$ locally, the change in link direction in $\tilde{\mathcal{P}}$ can be obtained by several "gauge transformations" of Kasteleyn orientation, which relate two different but equivalent Kasteleyn orientations (simultaneously changing the directions of links sharing a common vertex in $\tilde{\mathcal{P}}$ ) [65]. An example of the basic shape changes of singular lines on $\mathcal{T}$ and gauge transformation of Kasteleyn orientation on $\tilde{\mathcal{P}}$ is shown in Fig. 8. Note that the Majorana
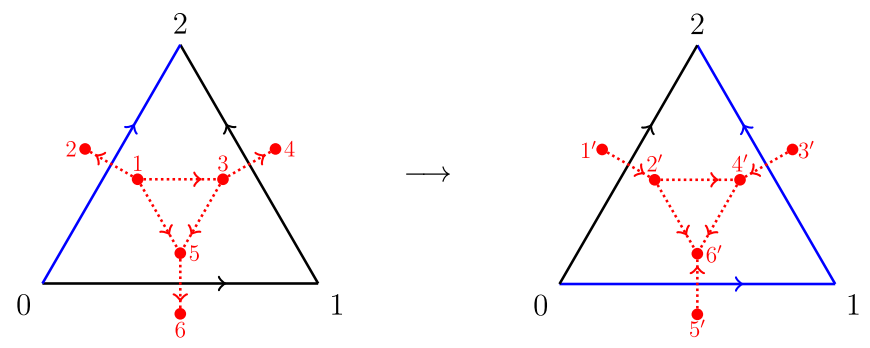

FIG. 8. Shape changing of singular lines $S$ of $\mathcal{T}$ and "gauge transformation" of Kasteleyn orientations of $\tilde{\mathcal{P}}$. We perform gauge transformations on the three red vertices inside a black triangle, which effectively change the shape of $S$ and the directions of the three outreaching red links. The Majorana fermions are mapped from $n$ to $n^{\prime}(n=1,2, \ldots, 6)$ with respect to the directions of red links dual to black links under this FSLU. degrees of freedom on the vertices of $\tilde{\mathcal{P}}$ are mapped from one lattice to another according to the link direction under the gauge transformation of Kasteleyn orientation ( $n$ to $n^{\prime}$ in Fig. 8). In this way, the vacuum state without fermions (without Kitaev chain) on the left lattice is mapped to the vacuum state on the right lattice without changing the fermion parity.

On the other hand, the homology class of $S$ matters. Different choices of topological classes of $S$ (fixed $w_{0}=\partial S$ ) correspond to different spin structures on $M$. Our constructions make sure that, for arbitrary choices of $S$, the local Kasteleyn properties along the smallest loop around every vertex are satisfied. However, the global Kasteleyn property along nontrivial cycles of $M$ can be either preserved or broken. They correspond to $2^{2 g}$ different spin structures on closed oriented surface $M$ with genus $g$ $[65,66]$. Different choices of $S$ induce different global Kasteleyn properties and thus correspond to different spin structures.

\section{Kasteleyn orientations under retriangulations}

In the above, we focus only on a fixed triangulation $\mathcal{T}$ of $M$ and relate its discrete Stiefel-Whitney homology class $\left[w_{0}\right]$ to the Kasteleyn orientations and spin structures. To use FSLU transformations to classify FSPT phases, we must understand the relation of Kasteleyn orientations for different triangulations. In fact, we only have to determine the changes of Kasteleyn orientations under Pachner moves, which are basic moves of retriangulation [71].

Ordinary Pachner moves for a two-dimensional manifold consist of a (2-2) move and a (1-3) move. With branching structures, there are three types of (2-2) move and four types of (1-3) move in total. (We do not consider the mirror images of these moves; otherwise, the number of moves would double.) Only two types of (2-2) move and two types of (1-3) move have branching structures that can be induced by global ordering [42]. Examples of these moves are shown in Figs. 9 and 10.

Other types of (2-2) and (1-3) moves are shown in Supplemental Material [72]. For Pachner moves that are not induced by global ordering, the representative $w_{0}$ of StiefelWhitney class $\left[w_{0}\right]$ in Eq. (19) may be changed. For Pachner moves that are induced by a global ordering, the representative $w_{0}$ of Stiefel-Whitney class $\left[w_{0}\right]$ is
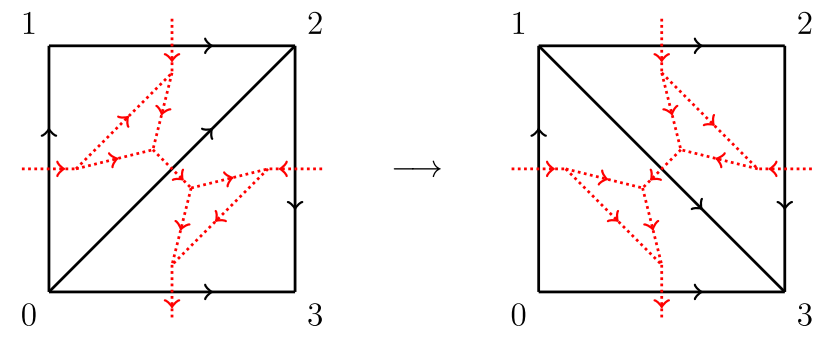

FIG. 9. Standard (2-2) move. 

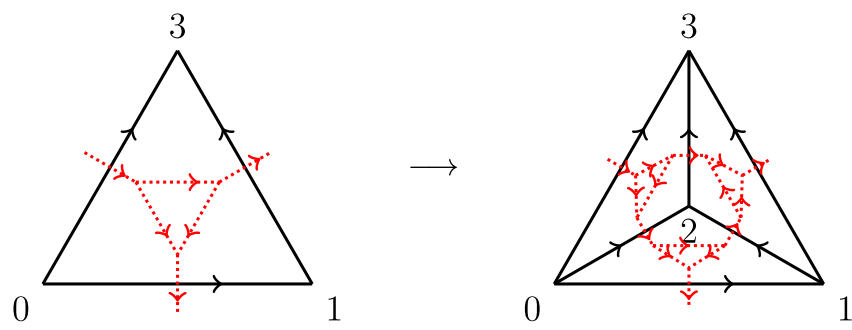

FIG. 10. One of the (1-3) moves.

unchanged. This makes the $2 \mathrm{D}$ case much easier than the 3D case.

\section{B. FSLU transformations and consistent conditions for fixed-point states}

In the above section, we discuss discrete spin structures and Kasteleyn orientation construction on arbitrary 2D triangulation lattices. We can now decorate Kitaev's Majorana chains using these rules and systematically classify 2D FSPT states by using FSLU transformations.

\section{Decoration of Kitaev's Majorana chains}

As discussed in Sec. II, our model has two types of fermionic degrees of freedom. The first type is the complex fermion $c_{(i j k)}$, which resides at the center of triangle $\langle i j k\rangle$ of space manifold triangulation $\mathcal{T}$. We use $n_{2}\left(g_{i}, g_{j}, g_{k}\right)=0$, 1 to denote the number of $c$ fermions at triangle $\langle i j k\rangle$. In fact, the parity conservation constraint for $c$ fermions under retriangulation is $d n_{2}=0(\bmod 2)$. Therefore, $n_{2}$ is an element of $H^{2}\left(G_{b}, \mathbb{Z}_{2}\right)$.

The second type of (complex) fermion, $a_{(i j)}$, resides on the link $\langle i j\rangle$ of $\mathcal{T}$. To describe Kitaev's Majorana chain more conveniently, we separate fermion $a_{(i j)}$ to two Majorana fermions:

$$
\begin{aligned}
& \gamma_{i j A}=a_{(i j)}+a_{(i j)}^{\dagger}, \\
& \gamma_{i j B}=\frac{1}{i}\left(a_{(i j)}-a_{(i j)}^{\dagger}\right) .
\end{aligned}
$$

The Majorana fermions $\gamma_{i j A}$ and $\gamma_{i j B}$ reside on the two sides of link $\langle i j\rangle$. They also reside on the two ends of the link in $\tilde{\mathcal{P}}$ dual to link $\langle i j\rangle$. Our convention is that the dual link has direction from vertex $\langle i j A\rangle$ to vertex $\langle i j B\rangle$. The fermion parity operator of $a$ fermions or $\gamma$ fermions at link $\langle i j\rangle$ is $P_{f}^{\gamma}=-i \gamma_{i j A} \gamma_{i j B}$.

Now we decorate Kitaev's Majorana chains onto the dual lattice. We use a $\mathbb{Z}_{2}$-valued 1-cochain $\tilde{n}_{1}\left(g_{i}, g_{j}\right)$ to indicate whether there is a domain wall between vertices $i$ and $j$. $\tilde{n}_{1} \in H^{1}\left(G_{b}, \mathbb{Z}_{2}\right)$ is a cocycle because we are constructing SPT states without deconfined Majorana fermions (the Kitaev chains should form closed loops). Depending on the configurations of $\left\{g_{i}\right\}$ and the choices of $\tilde{n}_{1}$, the domain wall configuration in a particular lattice is different. We pair the Majorana fermions depending on the domain wall configuration as follows. If there is no domain wall on link $\langle i j\rangle$, then the Majorana fermions $\gamma_{i j A}$ and $\gamma_{i j B}$ on the two sides of this link are paired (vacuum pair) with respect to the direction of the dual red link (we use a solid blue line and gray ellipse to indicate this pairing). If there is a domain wall on link $\langle i j\rangle$, then the Majorana fermion of this link is paired with another Majorana fermion belonging to another link with a domain wall within the same triangle.

After fermion decoration, the (2-2) move becomes a fermionic unitary transformation between the fermionic Fock spaces on two different triangulation lattices $\mathcal{T}$ and $\mathcal{T}^{\prime}$. An example of this $F$ move (the standard $F$ move) is presented as follows (there are $\mathbb{Z}_{2}$ domain walls on links $\langle 01\rangle,\langle 02\rangle,\langle 03\rangle$, and no domain wall on the other links):
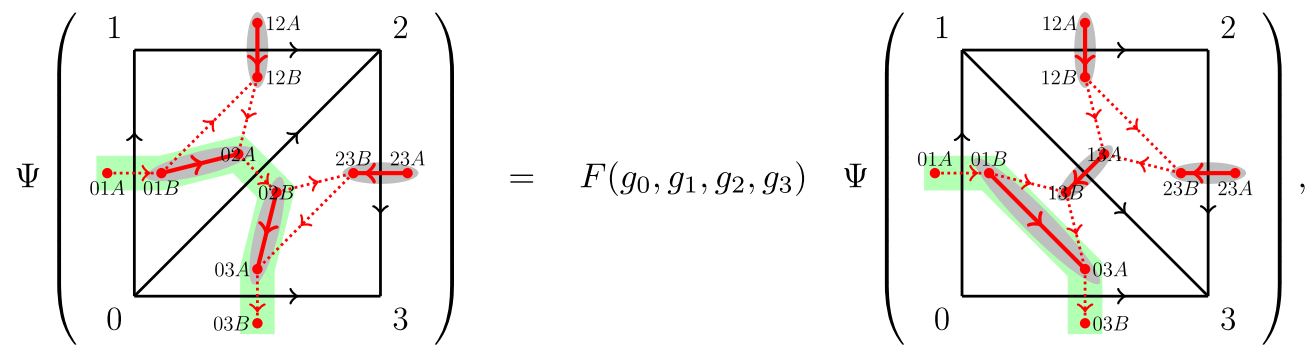

where the $F$ operator is defined as

$$
\begin{aligned}
F\left(g_{0}, g_{1}, g_{2}, g_{3}\right)= & \nu_{3}\left(g_{0}, g_{1}, g_{2}, g_{3}\right) c_{(012)}^{\dagger n_{2}\left(g_{0}, g_{1}, g_{2}\right)} c_{(023)}^{\dagger n_{2}\left(g_{0}, g_{2}, g_{3}\right)} \\
& \times c_{(013)}^{n_{2}\left(g_{0}, g_{1}, g_{3}\right)} c_{(123)}^{n_{2}\left(g_{1}, g_{2}, g_{3}\right)} X\left[\tilde{n}_{1}\left(g_{i}, g_{j}\right)\right] .
\end{aligned}
$$

Here, $\nu_{3}\left(g_{0}, g_{1}, g_{2}, g_{3}\right)$ is a $U_{T}(1)$-valued 3-cochain and $c_{(012)}^{\dagger}$ is the creation operator for $c$ fermions at triangle $\langle 012\rangle . X\left[\tilde{n}_{1}\left(g_{i}, g_{j}\right)\right]$ is a projection operator changing the Majorana fermion configurations. In the above example, the $X$ operator has an explicit form as follows:

$$
X\left[\tilde{n}_{1}\right]=2^{1 / 2}\left(P_{01 B, 02 A} P_{02 B, 03 A}\right) P_{13 A, 13 B},
$$

where $P_{a, b}=\left(1-i \gamma_{a} \gamma_{b}\right) / 2$ is the projection operator for Majorana pairs $\langle a, b\rangle$ (the direction is from vertex $a$ to 
vertex $b$ ). The first two projection operators in the above equation project the state to the Majorana dimer configuration in the left-hand figure. Note that the Majorana fermions, which do not appear explicitly on one lattice, are considered to be in vacuum pairs. For example, the two Majorana fermions $\gamma_{13 A}$ and $\gamma_{13 B}$ appear only in the righthand figure. They are considered to be paired from $\gamma_{13 A}$ to $\gamma_{13 B}$ in the left-hand figure. Therefore, we have a third projection operator in Eq. (25) to put the two Majorana fermions $\gamma_{13 A}$ and $\gamma_{13 B}$ into a vacuum state $\left(a_{(13)}^{\dagger} a_{(13)}=0\right)$ in the left-hand figure. All other Majorana fermions that are not shown in Eq. (25) are unchanged under the aforementioned $F$ move.

In order to make $X$ a unitary operator acting on the Hilbert space of Majorana fermions, we introduce a normalization factor in the front of $X$. By directly calculating the norm of the final state after the action of the $X$ operator, we can obtain a general expression of the normalization factor,

$$
\prod_{\operatorname{loop} i \text { in }\left(\tilde{\mathcal{P}}, \tilde{\mathcal{P}}^{\prime}\right)} 2^{\left(L_{i}-1\right) / 2},
$$

with $2 L_{i}$ being the length of the $i$ th loop in the transition graph of dimer configurations in $\tilde{\mathcal{P}}$ and $\tilde{\mathcal{P}}^{\prime}$. For example, the transition graph of the two states in Eq. (23) has only one loop with length bigger than two: $01 B-02 A-02 B-03 A-01 B$. So the factor is $2^{(4 / 2-1) / 2}=2^{1 / 2}$, according to Eq. (26).

As we are constructing FSPT states, the fermionic local unitary transformation $F$ should be $G_{b}$ symmetric in the sense that

$$
F\left(g_{0}, g_{1}, g_{2}, g_{3}\right)=F\left(g g_{0}, g g_{1}, g g_{2}, g g_{3}\right),
$$

for all $g \in G_{b}$ if $G_{b}$ is a unitary symmetry group. That is why $\nu_{3}\left(g_{0}, g_{1}, g_{2}, g_{3}\right), n_{2}\left(g_{0}, g_{1}, g_{2}\right)$, and $\tilde{n}_{1}\left(g_{0}, g_{1}\right)$ are all cochains that are invariant under unitary $g$ action. [We note that $\nu_{3}\left(g g_{0}, g g_{1}, g g_{2}, g g_{3}\right)=\nu_{3}^{*}\left(g_{0}, g_{1}, g_{2}, g_{3}\right)$ for antiunitary $g$ action.]

In general, there are eight kinds of domain wall configuration in the above $F$ move. One can show that for all configurations, the fermion parities of Majorana fermions are the same in the initial and final wave functions (this comes from the Kasteleyn orientation property of retriangulations; see Sec. III A and Supplemental Material [72]). Therefore, the fermion parities of $c$ fermions and $\gamma$ fermions should also be conserved separately, and both $n_{2}$ and $\tilde{n}_{1}$ are cocycles.

Similar to the 1D case, we can use FSLU to redefine the basis state $\left|\left\{g_{l}\right\}\right\rangle$ as

$$
\begin{aligned}
\left|\left\{g_{l}\right\}\right\rangle^{\prime}= & U_{\mu_{2}, m_{1}}\left|\left\{g_{l}\right\}\right\rangle \\
= & \prod_{\langle i j k\rangle} \mu_{2}\left(g_{i}, g_{j}, g_{k}\right)^{s\langle i j k\rangle} \prod_{\langle i j\rangle}\left[f_{i j A}^{m_{1}\left(g_{i}, g_{j}\right)} f_{i j B}^{m_{1}\left(g_{i}, g_{j}\right)}\right] \\
& \times \prod_{\langle i j k\rangle}\left[f_{j k A}^{\dagger m_{1}\left(g_{j}, g_{k}\right)} f_{i j A}^{\dagger m_{1}\left(g_{i}, g_{j}\right)} f_{i k B}^{\dagger m_{1}\left(g_{i}, g_{k}\right)}\right]\left|\left\{g_{l}\right\}\right\rangle,
\end{aligned}
$$

where we first create three complex fermions $f_{j k A}, f_{i j A}$, and $f_{i k B}$ near the three links of the triangle $\langle i j k\rangle(i<j<k)$, and then annihilate the two fermions $f_{i j A}$ and $f_{i j B}$ on the two sides of link $\langle i j\rangle$ when gluing the two triangles sharing link $\langle i j\rangle$. To preserve the fermion parity and be symmetric, $m_{1}$ should be a 1-cocycle (with $\mathbb{Z}_{2}$ coefficient): $m_{1}\left(g g_{i}, g g_{j}\right)=m_{1}\left(g_{i}, g_{j}\right)$ and $d m_{1}\left(g_{i}, g_{j}, g_{k}\right)=0$. $\mu_{2}\left(g_{i}, g_{j}, g_{k}\right)$ is a phase factor associated with triangle $\langle i j k\rangle$, and $s_{\langle i j k\rangle}= \pm 1$ denotes the orientation of the triangle. After eliminating all $f$ fermions in the new $F$ move operator $F^{\prime}=U_{\mu_{2}, m_{1}} F U_{\mu_{2}, m_{1}}^{\dagger}$ (all fermion signs are canceled again), one finds that the phase factor in Eq. (24) becomes

$$
\begin{aligned}
& \nu_{3}^{\prime}\left(g_{0}, g_{1}, g_{2}, g_{3}\right) \\
& \quad=\nu_{3}\left(g_{0}, g_{1}, g_{2}, g_{3}\right) \frac{\mu_{2}\left(g_{1}, g_{2}, g_{3}\right) \mu_{2}\left(g_{0}, g_{1}, g_{3}\right)}{\mu_{2}\left(g_{0}, g_{2}, g_{3}\right) \mu_{2}\left(g_{0}, g_{1}, g_{2}\right)},
\end{aligned}
$$

so the elements $\nu_{3}$ in the same group cohomology class in $H^{3}\left(G_{b}, U_{T}(1)\right)$ correspond to the same 2D FSPT phase. This is also consistent with the general result in Eq. (14) [42], since $S q^{2}\left(m_{1}\right)$ is also trivial in the 2D FSPT case.

Apart from the (2-2) move, there is another (2-0) move that can change the total number of vertices for triangulations. An example of domain wall configurations for the (2-0) move is

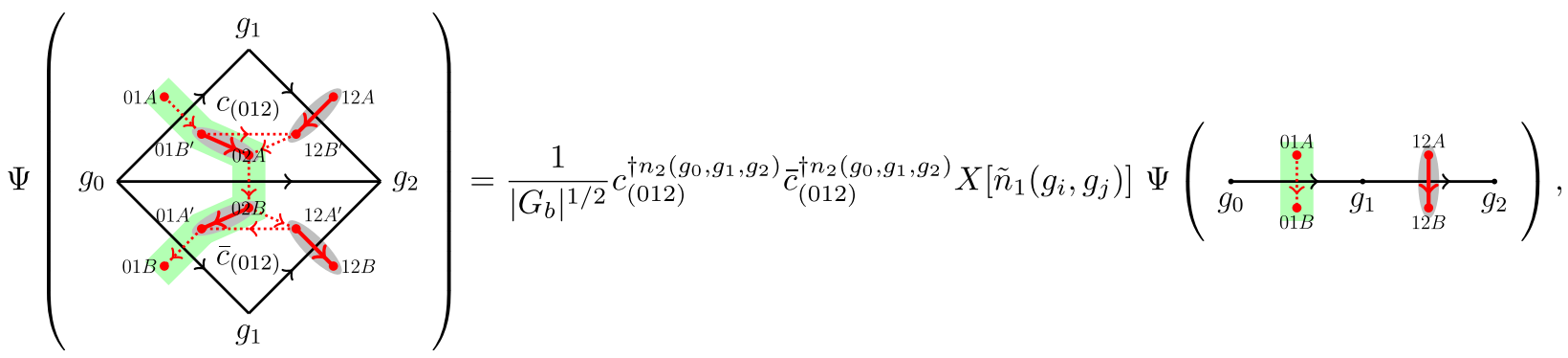


where $c_{(012)}$ and $\bar{c}_{(012)}$ are the annihilation operators of the $c$ fermions at the center of two triangles with opposite orientations in the left-hand figure $\left(_{(012)}\right.$ and $\bar{c}_{(012)}$ are at the centers of the upper and lower triangles in the left-hand figure, respectively). The Hilbert dimension of the bosonic degrees of freedom on the vertices of a fixed triangulation is $\left|G_{b}\right|^{N_{v}}$, where $\left|G_{b}\right|$ is the order of the group $G_{b}$ and $N_{v}$ is the number of vertices. Therefore, we add a normalization factor $\left|G_{b}\right|^{-1 / 2}$ in the front of the (2-0) move operator, for the vertex number is reduced by one from the left state to the right state. $X\left[\tilde{n}_{1}\right]$ is also the projection operator from the state of Majorana dimer pairs in the right-hand figure to the state of the left-hand figure. Note that there are six Majorana fermions $\left(\gamma_{02 A}, \gamma_{02 B}, \gamma_{01 A^{\prime}}, \gamma_{01 B^{\prime}}, \gamma_{12 A^{\prime}}\right.$, and $\left.\gamma_{12 B^{\prime}}\right)$ that do not appear explicitly in the right-hand figure. Similar to the case of the (2-2) move, these fermions should also be considered to be in vacuum pairs in the right figure state, such that $-i \gamma_{02 A} \gamma_{02 B}=-i \gamma_{01 B^{\prime}} \gamma_{01 A}=$ $-i \gamma_{12 B^{\prime}} \gamma_{12 A^{\prime}}=1$ when acting on the right figure state. This choice is possible because the dimer loop $01 A-01 B^{\prime}-01 A^{\prime}-01 B-01 A$ is Kasteleyn oriented. Therefore, one can also use the convention that the two Majorana fermions on the two sides of a link are paired up by regarding the projection operators $-i \gamma_{01 A} \gamma_{01 B^{\prime}},-i \gamma_{01 A^{\prime}} \gamma_{01 B}$, $-i \gamma_{12 A} \gamma_{12 B^{\prime}}$, and $-i \gamma_{12 A^{\prime}} \gamma_{12 B}$ as 1 when acting on the vacuum state of the left-hand figure. The $X$ operator then projects the state to the Majorana dimer configuration state in the left-hand figure. The fermion parities of the left and right states are always the same. An explicit expression of $X$ for this particular (2-0) move is

$$
X\left[\tilde{n}_{1}\right]=2 P_{01 B^{\prime}, 02 A} P_{02 B, 01 A^{\prime}} P_{12 A, 12 B^{\prime}} P_{12 A^{\prime}, 12 B} .
$$

Using the (2-0) moves, we can deduce all (3-1) moves and other (2-2) moves from the standard (2-2) $F$ move in Eq. (23). The normalization factor is obtained from Eq. (26). There are two loops in the transition graph of the Majorana dimer states with length bigger than two: $01 B^{\prime}-02 A-02 B-01 A^{\prime}-01 B^{\prime}$ and $12 A-12 B^{\prime}-12 A^{\prime}-12 B-12 A$. So the normalization factor is $2^{(4 / 2-1) / 2} \times 2^{(4 / 2-1) / 2}=2$.

\section{Fermionic pentagon equations}

In the above, we discuss the FSLU moves. The most important one is the standard $F$ move in Eq. (23). Similar to the bosonic pentagon equation for the bosonic $F$ move, we have a fermionic pentagon equation as a consistent equation for FSLU transformations (see Fig. 11). This fermionic pentagon equation involves only the standard $F$ move. Using the unitary conditions, one can also derive other pentagon equations, and they essentially give the same constraint for $\nu_{3}$.

We now calculate the constraint for $\nu_{3}$ from the pentagon equation in Fig. 11. As the $c$ fermions and Majorana fermions are decoupled in the $F$ move [the $c$ fermion part and the Majorana fermion part of $X$ in Eq. (24) commute], only the $c$ fermion twists the cocycle condition for $\nu_{3}$. The $X$ operators are merely projection operators that do not introduce any nontrivial phases in two different paths of pentagon equation. The final result of the equation for $\nu_{3}$ is the same as (special) group supercohomology theory $[42,63]$; i.e.,

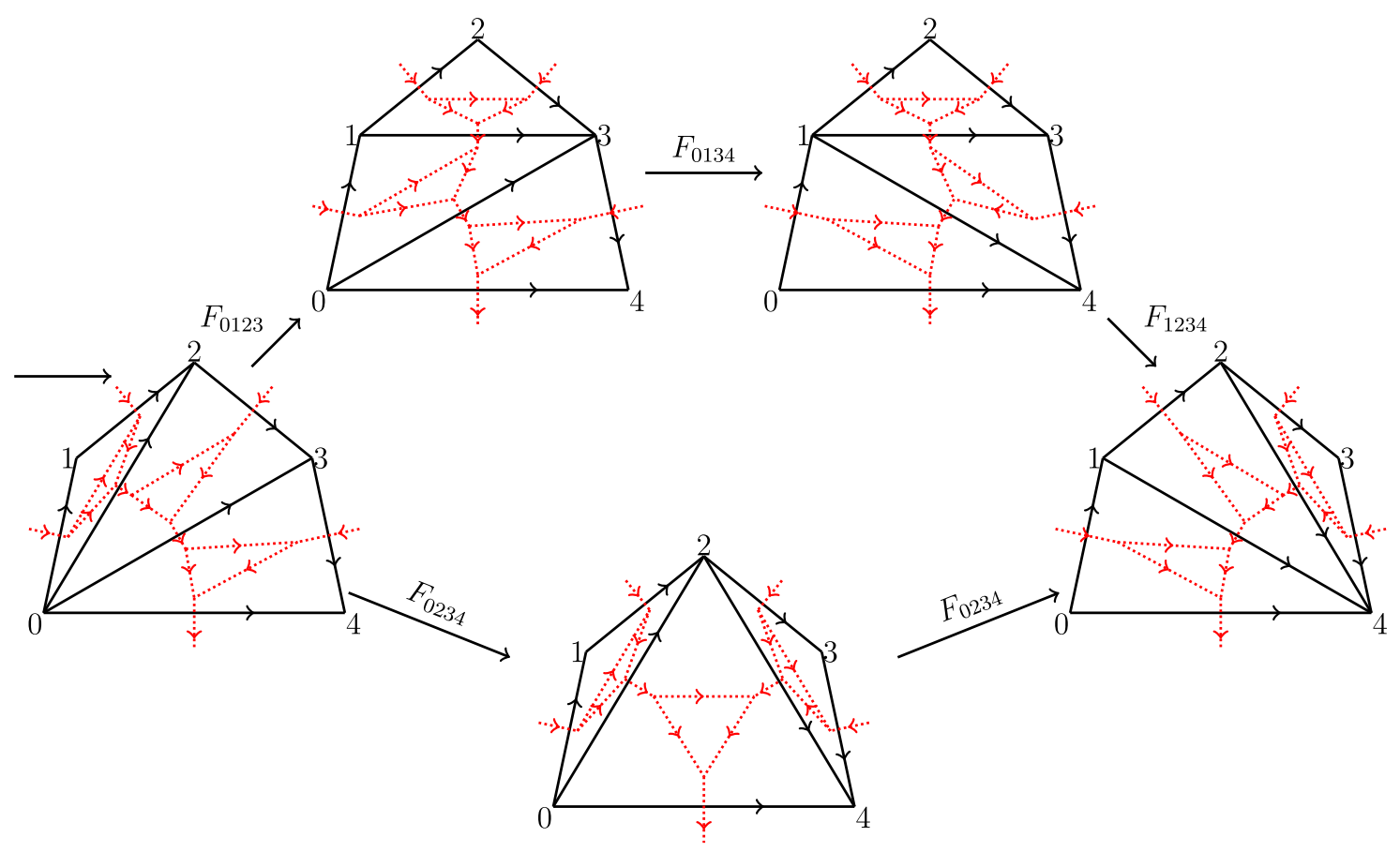

FIG. 11. Fermionic pentagon equation. 


$$
\begin{aligned}
\left(d \nu_{3}\right)\left(g_{0}, g_{1}, g_{2}, g_{3}, g_{4}\right) & =(-1)^{S q^{2}\left(n_{2}\right)\left(g_{0}, g_{1}, g_{2}, g_{3}, g_{4}\right)} \\
& =(-1)^{n_{2}\left(g_{0}, g_{1}, g_{2}\right) n_{2}\left(g_{2}, g_{3}, g_{4}\right)} .
\end{aligned}
$$

Now, we see that only $B H^{2}\left(G_{b}, \mathbb{Z}_{2}\right)$, the obstruction-free subgroup of $H^{2}\left(G_{b}, \mathbb{Z}_{2}\right)$ formed by elements $n_{2} \in$ $H^{2}\left(G_{b}, \mathbb{Z}_{2}\right)$ that satisfy $S q^{2}\left(n_{2}\right)=0$ in $H^{4}\left(G_{b}, U_{T}(1)\right)$, can give rise to solutions for $\nu_{3}$, and inequivalent solutions of $\nu_{3}$ are still given by $H^{3}\left(G_{b}, U_{T}(1)\right)$ according to the gauge transformations of $\nu_{3}$. Thus, the mathematical objects that classify 2D FSPT phases with a total symmetry $G_{f}=G_{b} \times \mathbb{Z}_{2}^{f}$ can be summarized as three group cohomologies of the symmetry group $G_{b}[48,50]: H^{1}\left(G_{b}, \mathbb{Z}_{2}\right)$, $B H^{2}\left(G_{b}, \mathbb{Z}_{2}\right)$, and $H^{3}\left(G_{b}, U_{T}(1)\right)$. Finally, by using the method proposed in Ref. [63], one can derive the commuting projector parent Hamiltonian of all of these FSPT states on arbitrary 2D triangulations with a branching structure.

\section{CONSTRUCTIONS AND CLASSIFICATIONS FOR FSPT STATES IN 3D}

In this section, we construct and classify the 3D FSPT states parallel to the discussions of 2D FSPT states. Compared with the 2D case, the most nontrivial part of 3D phases is the fermion parity mixing of the $c$ fermions and Majorana fermions. In Sec. IV A, we find that there are in general no Kasteleyn orientations on a 3D lattice. The existence of a spin structure only implies local Kasteleyn orientations. If we decorate Kitaev's Majorana chains onto a 3D lattice, the shape-changing process of the Majorana chain may also change the fermion parity of the corresponding Majorana chain. In this case, we should use the $c$ fermion to compensate the fermion parity changes. As a result of this fermion parity mixing, the cocycle equation for $\nu_{4}$ is much more complicated than special group supercohomology theory.

\section{A. Discrete spin structure in 3D and local Kasteleyn orientations}

In this section, we discuss the first Stiefel-Whitney homology class on a discrete lattice and relate it to the local Kasteleyn orientations on the dual lattice. The overall constructions are parallel to the 2D case. The difference is that Kasteleyn orientations are only satisfied for the smallest loops in 3D, not for the general large loops. The fermion parity of a Kitaev chain decorated onto a fluctuating loop is therefore not conserved.

\section{Discrete Stiefel-Whitney homology class $w_{1}$}

Similar to the oriented 2D manifolds, all oriented 3D manifolds admit spin structures. The second StiefelWhitney cohomology class $\left[w^{2}\right]$ is always trivial. We can consider the first discrete Stiefel-Whitney homology class for a triangulation $\mathcal{T}$ with a branching structure of $3 \mathrm{D}$ spatial manifold $M$ [69]:

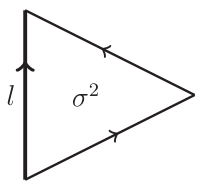

(a) $\sigma^{1}=l$ is an 1-simplex.

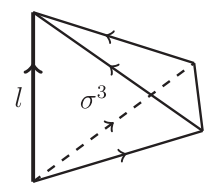

(c) $\sigma^{3}$ is a 3 -simplex.
FIG. 12. Regular pairs $l \subseteq \sigma^{i}(i=1,2,3)$ for link $l$.

$$
w_{1}=\sum_{l \in \mathcal{T}} \mathcal{N}\{\sigma \mid l \subseteq \sigma \text { is regular }\} l
$$

$w_{1}$ is the summation of all links in $\mathcal{T}$ with some $\mathbb{Z}_{2}$ coefficients. Again, $l \subseteq \sigma$ means that link $l$ is a subsimplex of simplex $\sigma . l \subseteq \sigma$ is called regular if $l$ and $\sigma$ have one of the three relative positions shown in Fig. 12. If $\mathcal{N}\{\sigma \mid l \subseteq \sigma$ is regular $\}$ is odd, we call the link $l$ singular. Thus, $w_{1}$ in Eq. (33) is the formal summation of all singular lines. $w_{1}$ is a vector (1st singular chain) in the vector space (of 1th singular chains) spanned by formal bases of all links with $\mathbb{Z}_{2}$ coefficients.

As the second Stiefel-Whitney cohomology class $\left[w^{2}\right]$ of any oriented 3D manifold is trivial, we can find some surface $S$ such that $w_{1}=\partial S$. For a fixed collection of singular links (a fixed representative $w_{1}$ for $\left[w_{1}\right]$ ), different inequivalent choices of $S$ correspond to different spin structures (see the discussions at the end of Sec. III A 1).

\section{Local Kasteleyn orientations and gauge transformations}

In 3D, we also want to decorate Kitaev chains onto the intersection lines of $G_{b}$-symmetry domain walls. A natural question inherited from 2D is whether there are Kasteleyn properties for all even-link loops in 3D. This question is related to the fermion parity of the Kitaev chain. The answer is that the existence of discrete spin structures (the vanishing of $\left.\left[w_{1}\right]\right)$ is related to the existence of local Kasteleyn orientations of the resolved dual lattice. In other words, Kasteleyn properties are satisfied for the smallest loops but generally broken for large loops in 3D.

We now consider the construction that is similar to the $2 \mathrm{D}$ case. For a fixed triangulation $\mathcal{T}$ of $3 \mathrm{D}$ manifold $M$, the first step is to construct a polyhedral decomposition $\mathcal{P}$ of $M$, which is a 4-valent graph dual to $\mathcal{T}$. We now add a spinless fermionic degree of freedom to every face (triangle) of $\mathcal{T}$ and split it into two Majorana fermions on the two sides of this face for convenience. Equivalently, we resolve the triangulation $\mathcal{T}$ by adding a new vertex to each tetrahedron center and obtain a new resolved triangulation $\tilde{\mathcal{T}}$. The Majorana fermions reside on the vertices of the resolved dual lattice $\tilde{\mathcal{P}}$, which is a 4 -valent graph dual to $\tilde{\mathcal{T}}$ (see Fig. 13).

The second step is again adding directions to links in $\mathcal{T}$ and $\tilde{\mathcal{P}}$. The directions of the links in $\mathcal{T}$ are given by the 


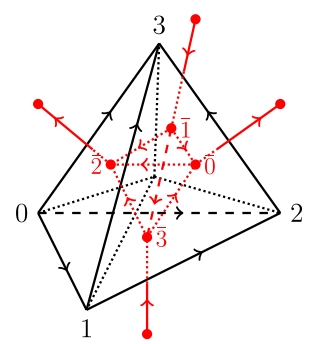

(a) Positive oriented tetrahedron

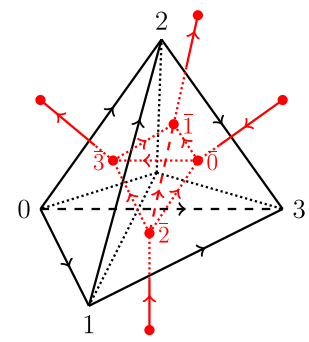

(b) Negative oriented tetrahedron
FIG. 13. Triangulation $\mathcal{T}$ (solid black line), resolved triangulation $\tilde{\mathcal{T}}$ (solid and dashed black lines), and resolved dual lattice $\tilde{\mathcal{P}}$ (red line). The resolved triangulation $\tilde{\mathcal{T}}$ is obtained from the original $\mathcal{T}$ by adding a new vertex to the center of each tetrahedron. The links of $\mathcal{P}$ have orientations induced from the link orientations of $\mathcal{T}$ according to the conventions shown in Fig. 14(a). Red dots on the vertices of $\tilde{\mathcal{P}}$ represent Majorana fermions, which are split from complex fermions on each face of $\mathcal{T}$.

branching structure. The dual link direction in $\mathcal{P}$ is obtained from $\mathcal{T}$ using the convention shown in Fig. 14(a). The directions of the new links in $\tilde{\mathcal{P}}$ are obtained from the triangulation $\mathcal{T}$ by using the conventions in Fig. 13 .

The above link direction construction has the following properties. Consider a fixed link $l \in \mathcal{T}$. When going along the smallest red loop in $\tilde{\mathcal{P}}$ around this link $l$ along the righthand rule direction, we encounter an even number of red links with directions along or opposite to our direction. Using the conventions in Fig. 13, the red link direction is opposite to our direction if and only if (1) the red link is dual to a black triangle in $\mathcal{T}$ such that the initial and final vertices of $l$ are the first and last vertices of this black triangle [this corresponds to the case in Fig. 12(b)] and (2) the red link is a resolved new link inside a tetrahedron in $\mathcal{T}$ such that the initial and final vertices of $l$ are the first and the last vertices of this tetrahedron [this is the case in Fig. 12(c)]. If the total number of red links with opposite directions is odd, we call the link $l$ Kasteleyn oriented. Under this construction, we relate the first Stiefel-Whitney homology class $w_{1}$ in Eq. (33) to the orientations of links in

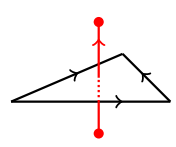

(a) Orientation convention for (red) link dual to (black) triangle $f \notin S$.

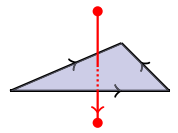

(b) Orientation convention for (red) link dual to (blue) singular triangle $f \in S$.
FIG. 14. Conventions for orientations of links in $\tilde{\mathcal{P}}$ (red line) from branching structure of triangulation $\mathcal{T}$ (black line). Nonsingular (singular) black (blue) triangle $f \notin S(f \in S)$ induces orientation for the dual link in $\tilde{\mathcal{P}}$. We introduce a spinless fermion on each (black or blue) triangle in $\mathcal{T}$ and split it into two Majorana fermions on two sides of this triangle or vertices of $\tilde{\mathcal{P}}$ (red dots).
$\tilde{\mathcal{P}}$; i.e., $w_{1}$ is the summation of all non-Kasteleyn-oriented links.

$$
\begin{aligned}
w_{1}= & \sum_{l \in \mathcal{T}}\left(1+\mathcal{N}\left\{\sigma^{2} \mid l \subseteq \sigma^{2} \text { is regular }\right\}\right. \\
& \left.+\mathcal{N}\left\{\sigma^{3} \mid l \subseteq \sigma^{3} \text { is regular }\right\}\right) \cdot l \\
= & \sum_{l \in \mathcal{T}} l(l \text { is non-Kasteleyn oriented }) .
\end{aligned}
$$

As discussed above, the first Stiefel-Whitney homology class $\left[w_{1}\right]$ for any oriented 3D manifold is trivial. Therefore, we have some singular surface $S$ such that $w_{1}=\partial S$. Now, if we reverse the direction of the links in $\tilde{\mathcal{P}}$ crossing the singular surface $S$, as shown in Fig. 14(b), then all of the links in $\mathcal{T}$ are Kasteleyn oriented. After following the procedures above, we relate the vanishing of the zeroth Stiefel-Whitney homology class $\left[w_{0}\right]$ to the property of local Kasteleyn orientation of the smallest loops around all of the links in $\mathcal{T}$. Here, "local" means that only the smallest loops in $\tilde{\mathcal{P}}$ around links in $\mathcal{T}$ are Kasteleyn oriented. Larger loops with an even number of links do not have Kasteleyn properties in general.

The above construction of link directions in $\tilde{\mathcal{P}}$ depends on the choice of singular surface $S$. The shape of $S$ can also be changed with fixed $\partial S$. If we change the shape of $S$ locally, the changes of link directions in $\tilde{\mathcal{P}}$ can be obtained by several "gauge transformations" of Kasteleyn orientations. We define this by simultaneously changing the directions of links sharing a common vertex in $\tilde{\mathcal{P}}$, similar to the 2D case [65]. Different Kasteleyn orientations related by these gauge transformations are said to be equivalent. An example of shape changes of singular surfaces on $\mathcal{T}$ and gauge transformation of Kasteleyn orientations on $\tilde{\mathcal{P}}$ are shown in Fig. 15. The Majorana degrees of freedom on the vertices of $\tilde{\mathcal{P}}$ are also mapped from one lattice to another
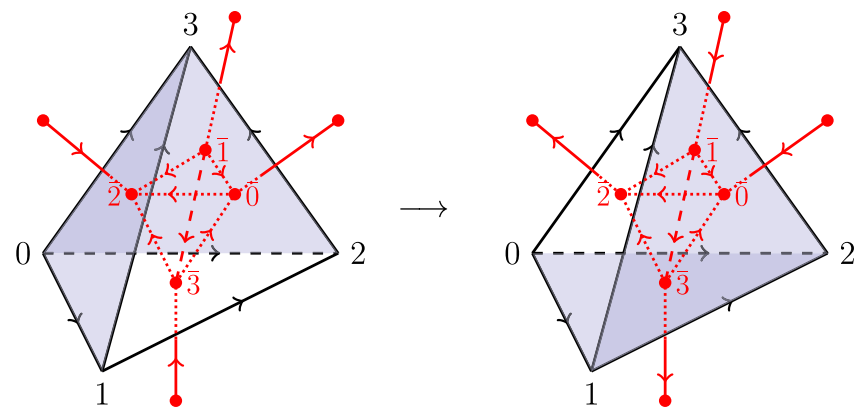

FIG. 15. Shape changing of singular surfaces $S$ of $\mathcal{T}$ and gauge transformation of Kasteleyn orientations of $\tilde{\mathcal{P}}$. We perform gauge transformations on the four red vertices inside a black tetrahedron, which effectively changes the shape of $S$ and the directions of the four outreaching red links. The Majorana fermions are mapped with respect to the directions of red links dual to black triangles under this FSLU transformation. 
according to the link directions (similar to the 2D case in Fig. 8). This ensures that the vacuum state (without Kitaev chain) on one lattice is mapped to the vacuum state on another lattice, without fermion parity changing (no fermion on either lattice).

With fixed $w_{0}=\partial S$, the choices of $S$ are not unique. Different choices of topological classes of $S$ correspond to different spin structures on $M$. The global Kasteleyn properties along nontrivial cycles of $M$ can also be either preserved or broken depending on the choices of $S$. Our construction generalizes the relation of Kasteleyn orientations and discrete spin structures from 2D [65] to $3 \mathrm{D}$.

\section{Local Kasteleyn orientations under retriangulations}

To perform FSLU transformations, we now consider that the Kasteleyn orientation changes under retriangulation of
$M$. Pachner moves for the 3D manifold consist of a (2-3) move and a (1-4) move [71]. When introducing branching structures, there are 10 types of (2-3) move and 5 types of (1-4) move (again, we do not consider the mirror images of these moves, otherwise the number would double) [42]. Eight types of (2-3) move and three types of (1-4) move have branching structures that can be induced by global ordering [42,73]. The standard (2-3) move is given in Fig. 16, which does not involve singular surfaces. Two examples of moves involving singular surfaces [the representative $w_{1}$ of Stiefel-Whitney class $\left[w_{1}\right]$ in Eq. (33) is changed] are presented in Figs. 17 and 18. Other types of (2-3) and (1-4) moves are shown in Supplemental Material [72].

Until now, the construction in 3D has been very similar to the 2D case. However, there is a very crucial difference. Although both the lattices before and after the Pachner move have local Kasteleyn properties, the Kasteleyn orientation for larger loops may be broken. Consider,

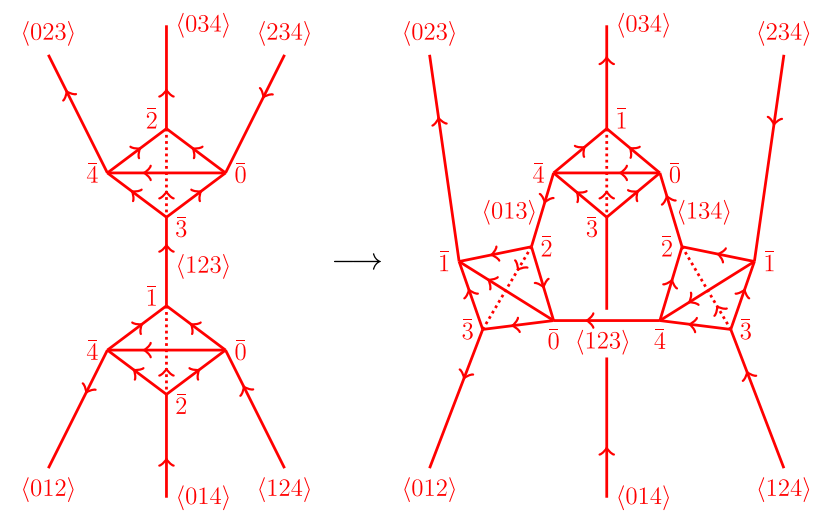

(b) For resolved dual lattice $\tilde{\mathcal{P}}$.

FIG. 16. Standard (2-3) move.
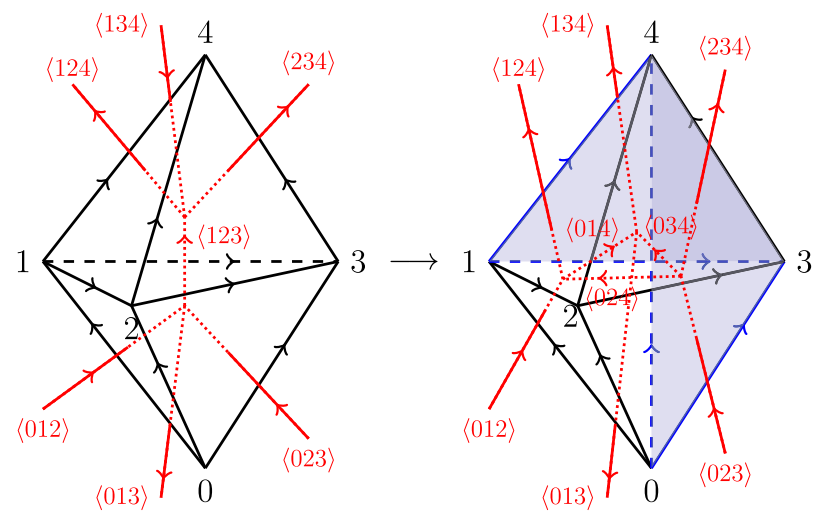

(a) (2-3) move for triangulation $\mathcal{T}$ and dual lattice $\mathcal{P}$. Singular links $w_{1}=\langle 14\rangle+\langle 34\rangle+\langle 03\rangle+\langle 04\rangle$ and surfaces $S=\langle 034\rangle+\langle 134\rangle$ are represented by blue lines and blue triangles, respectively.

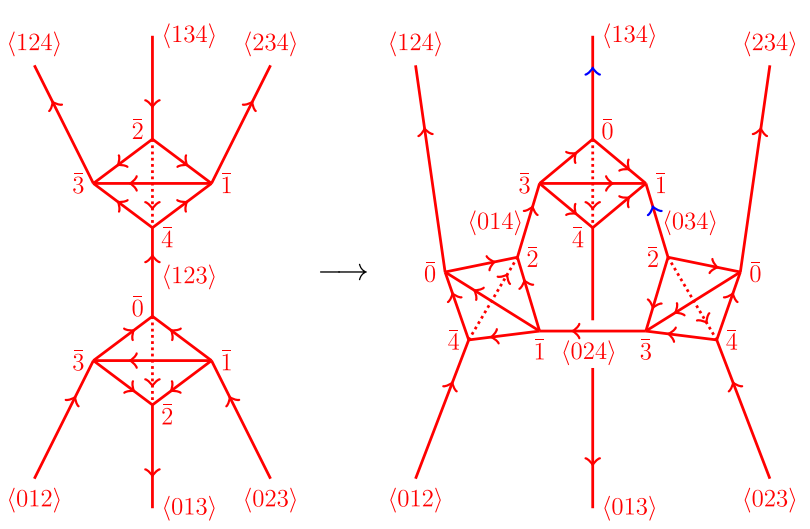

(b) (2-3) move for resolved dual lattice $\tilde{\mathcal{P}}$. Blue arrows on dual (red) links $\langle 034\rangle$ and $\langle 134\rangle$ indicate that the directions are reversed by singular surfaces $S$.

FIG. 17. A (2-3) move that involves singular surfaces. 


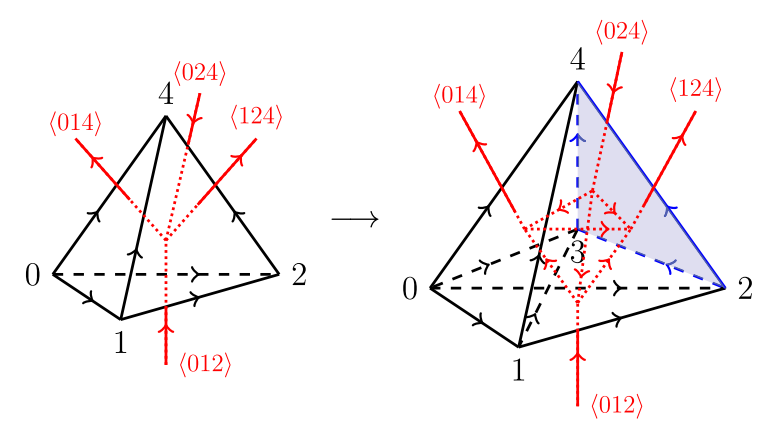

(a) (1-4) move for triangulation $\mathcal{T}$ and dual lattice $\mathcal{P}$ Singular links $w_{1}=\langle 23\rangle+\langle 34\rangle+\langle 24\rangle$ and surfaces $S=\langle 234\rangle$ are represented by blue lines and blue triangles, respectively.

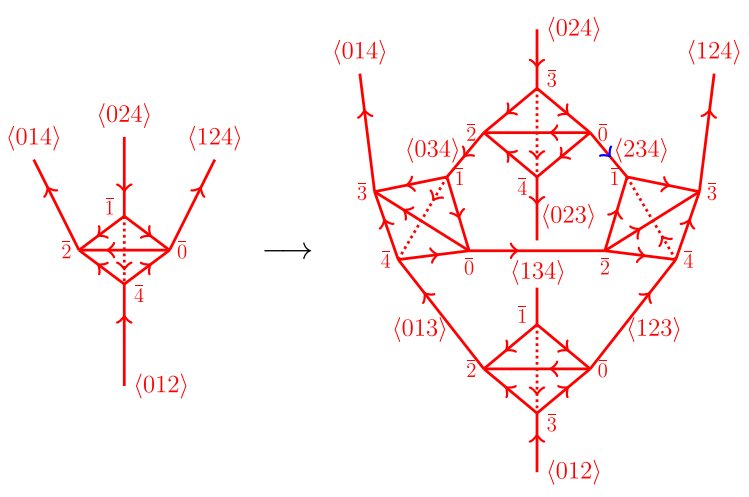

(b) (1-4) move for resolved dual lattice $\tilde{\mathcal{P}}$. Blue arrow on dual (red) links $\langle 234\rangle$ indicates that the directions are reversed by singular surfaces $S$.

FIG. 18. A (1-4) move.

for example, the case in Fig. 17. If we consider that the large loop consists of links $\langle 012\rangle,\langle 123\rangle$, and $\langle 124\rangle$ (and the newly resolved links between them) on the left lattice $\tilde{\mathcal{P}}$ and links $\langle 012\rangle$ and $\langle 124\rangle$ (and the newly resolved links between them) on the right lattice $\tilde{\mathcal{P}}^{\prime}$, the Kasteleyn properties are the same (all five links on $\tilde{\mathcal{P}}$ and three links on $\tilde{\mathcal{P}}^{\prime}$ have an up direction). On the other hand, if we consider that the large loop consists of links $\langle 012\rangle,\langle 123\rangle$, and $\langle 234\rangle$ on the left lattice $\tilde{\mathcal{P}}$ and links $\langle 012\rangle,\langle 024\rangle$, and $\langle 234\rangle$ on the right lattice $\tilde{\mathcal{P}}^{\prime}$, the Kasteleyn properties are changed (all five links on $\tilde{\mathcal{P}}$ have an up direction, but only two of five links on $\tilde{\mathcal{P}}^{\prime}$ have an up direction). In the next section, we systematically analyze the Kasteleyn properties of loops under Pachner moves. After decorating Majorana fermions, we see that the Majorana fermion parity changes, which is crucial in constructing legitimate FSPT states.

\section{B. FSLU transformations and consistent conditions for fixed-point states}

With the above setup of discrete spin structures and Kasteleyn orientation construction on a 3D lattice, we can now use the FSLU transformation to classify 3D FSPT states systematically.

\section{Fermion parity conservation and the obstruction of Kitaev's Majorana chain decoration}

Similar to the 2D case, our 3D model has two types of fermionic degrees of freedom. The first type is the complex fermion $c_{(i j k l)}$, which resides at the center of tetrahedron $\langle i j k l\rangle$ of triangulation $\mathcal{T}$ of the space manifold. In the special group supercohomology wave function, the $c$ fermion parity $P_{f}^{c}$ is unchanged under (2-3) and (1-4) moves. If we use $n_{3}\left(g_{i}, g_{j}, g_{k}, g_{l}\right)=0,1$ to denote the number of $c$ fermions at tetrahedron $\langle i j k l\rangle$, then the parity conserved condition becomes $d n_{3}=0(\bmod 2)$. Therefore, $n_{3}$ is an element of $H^{3}\left(G_{b}, \mathbb{Z}_{2}\right)$. This is not true if we introduce the second type of fermion.

The second type of fermion, complex fermion $a_{(i j k)}$, resides on triangle $\langle i j k\rangle$ of $\mathcal{T}$. Similar to the $2 \mathrm{D}$ case, we also separate fermion $a_{(i j k)}$ into two Majorana fermions:

$$
\begin{aligned}
& \gamma_{i j k A}=a_{(i j k)}+a_{(i j k)}^{\dagger}, \\
& \gamma_{i j k B}=\frac{1}{i}\left(a_{(i j k)}-a_{(i j k)}^{\dagger}\right) .
\end{aligned}
$$

The Majorana fermions $\gamma_{i j k A}$ and $\gamma_{i j k B}$ reside on the two sides of triangle $\langle i j k\rangle$, or dually, on two ends of the link in $\tilde{\mathcal{P}}$ dual to triangle $\langle i j k\rangle$. Our convention is that the dual link (we also use $\langle i j k\rangle$ to denote the dual link) has direction from vertex $\langle i j k A\rangle$ to $\langle i j k B\rangle$. As such, the fermion parity operator of $a$ fermion or $\gamma$ fermion at triangle $\langle i j k\rangle$ is $P_{f}^{\gamma}=-i \gamma_{i j k A} \gamma_{i j k B}$.

Now we decorate Kitaev's Majorana chains onto the loops in dual lattice $\mathcal{P}$. We introduce a $\mathbb{Z}_{2}$ cochain $\tilde{n}_{2}\left(g_{i}, g_{j}, g_{k}\right)=0,1$ to specify the decoration configuration of Kitaev's Majorana chain. If there is a Kitaev chain that goes though link $\langle i j k\rangle$ in $\mathcal{P}$ [see the green links in Fig. 3 and figures in Eq. (40)], then we set $\tilde{n}_{2}\left(g_{i}, g_{j}, g_{k}\right)=1$. On the other hand, $\tilde{n}_{2}\left(g_{i}, g_{j}, g_{k}\right)=0$ means there is no Kitaev chain. The Kitaev chain decorations can be translated to dimer configurations of Majorana pairs in the resolved dual lattice $\tilde{\mathcal{P}}$. $\tilde{n}_{2}\left(g_{i}, g_{j}, g_{k}\right)=0$ indicates vacuum pairing; i.e., the two Majorana fermions at triangle $\langle i j k\rangle$ are paired up from $\langle i j k A\rangle$ to $\langle i j k B\rangle$. If $\tilde{n}_{2}\left(g_{i}, g_{j}, g_{k}\right)=1$, then $\gamma_{i j k A}$ and $\gamma_{i j k B}$ are paired up with other nearby Majorana fermions separately, similar to the construction of Kitaev's Majorana chain [see figures of Eq. (41), where gray ellipses indicate paired Majorana fermions]. 


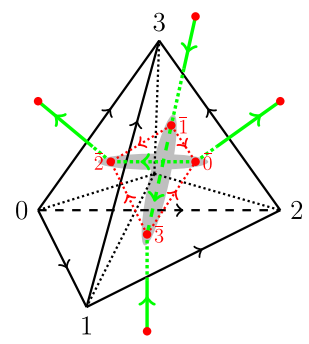

(a) Positive oriented tetrahedron

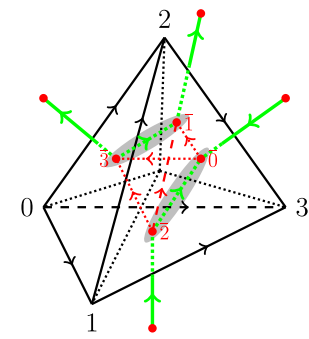

(b) Negative oriented tetrahedron

FIG. 19. Resolvation of four strings of Kitaev's Majorana chains meeting at one tetrahedron. If four strings meet at one tetrahedron, we should pair the Majorana fermions $\overline{0}$ to $\overline{2}$ and $\overline{1}$ to $\overline{3}$, respectively (gray ellipses). The green lines on $\tilde{\mathcal{P}}$ indicate that these lines are decorated by Kitaev chains.

As we are constructing a SPT state without intrinsic anyonic excitations, the decorated Kitaev chain should form a closed loop without ends. Therefore, similar to the 2D case, we have the equation $d \tilde{n}_{2}=0(\bmod 2)$, which means that the cochain $\tilde{n}_{2}$ is an element of $H^{2}\left(G_{b}, \mathbb{Z}_{2}\right)$. It is possible that all four faces of a tetrahedron $\langle 0123\rangle$ in $\mathcal{T}$ are decorated with Kitaev chains; i.e., $d \tilde{n}_{2}\left(g_{0}, g_{1}, g_{2}, g_{3}\right)=4$. There are ambiguities in pairing four Majorana fermions inside the tetrahedron. For the total three possible pairings, we use the convention that the Majorana fermion $\overline{0}$ is paired to $\overline{2}$ and $\overline{1}$ is paired to $\overline{3}$ (see Fig. 19). One can also choose other conventions which essentially produce the same results [74].

Now we turn to the Pachner moves for different triangulations. We find that the Majorana fermion parity is changed under a (2-3) move if and only if

$$
\begin{aligned}
S q^{2}\left(\tilde{n}_{2}\right)\left(g_{0}, g_{1}, g_{2}, g_{3}, g_{4}\right) & =\tilde{n}_{2}\left(g_{0}, g_{1}, g_{2}\right) \tilde{n}_{2}\left(g_{2}, g_{3}, g_{4}\right) \\
& =1 .
\end{aligned}
$$

We use the notation $S q^{2}\left(\tilde{n}_{2}\right)=\tilde{n}_{2}^{2}=\tilde{n}_{2} \smile \tilde{n}_{2}$ later. This can be obtained by directly checking the property of Kasteleyn orientation for the loops in the transition graph of two Majorana dimer states for 64 kinds of string configurations of each (2-3) move (see Supplemental Material for details [72]). To compensate for the Majorana fermion parity changes, the fermion parity of the fermions at the center of tetrahedron should also be changed, leading to the $(\bmod 2)$ equation

$$
d n_{3}=\tilde{n}_{2} \smile \tilde{n}_{2},
$$

or more explicitly,

$$
\begin{aligned}
n_{3}\left(g_{1}, g_{2}, g_{3}, g_{4}\right)+n_{3}\left(g_{0}, g_{2}, g_{3}, g_{4}\right)+n_{3}\left(g_{0}, g_{1}, g_{3}, g_{4}\right) \\
\quad+n_{3}\left(g_{0}, g_{1}, g_{2}, g_{4}\right)+n_{3}\left(g_{0}, g_{1}, g_{2}, g_{3}\right) \\
\quad=\tilde{n}_{2}\left(g_{0}, g_{1}, g_{2}\right) \tilde{n}_{2}\left(g_{2}, g_{3}, g_{4}\right) .
\end{aligned}
$$

The above equation shows that the cocycle equation of $n_{3}$ is twisted by $\tilde{n}_{2}^{2}$, which is different from $d n_{3}=0$ in the special group supercohomology model [42]. The above equation for $n_{3}$ has solutions if and only if $S q^{2}\left(\tilde{n}_{2}\right)$ is the trivial element in $H^{4}\left(G_{b}, \mathbb{Z}_{2}\right)$.

\section{Fermionic symmetric local unitary transformations}

After fermion decoration, the standard (2-3) move Fig. 16 becomes a fermionic unitary transformation between the fermionic Fock spaces on two different triangulation lattices $\mathcal{T}$ (left) and $\mathcal{T}^{\prime}$ (right). An example of this standard $F$ move, which changes the fermion parity of Majorana fermions, is [on lattice $\mathcal{T}, \mathcal{P}$ in Eq. (40) and on $\tilde{\mathcal{P}}$ in Eq. (41)]

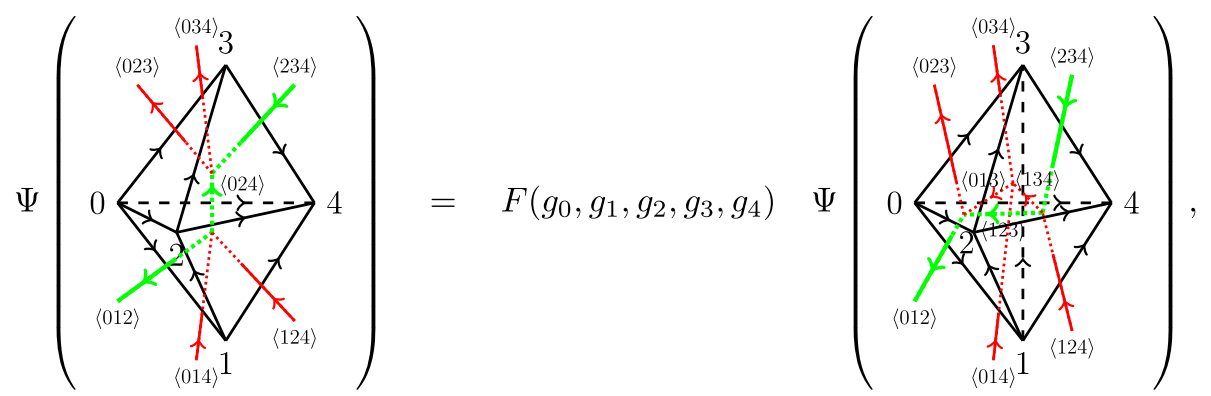




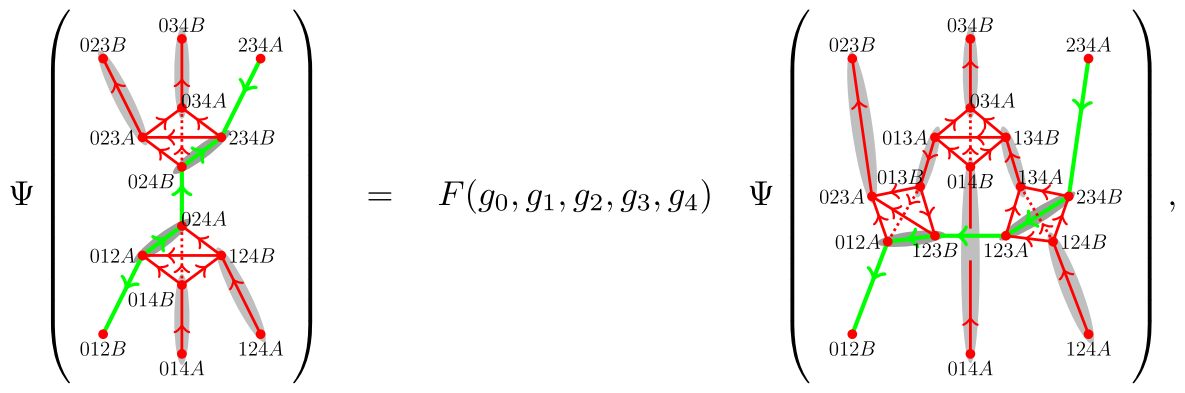

where the $F$ operator is given by

$$
\begin{aligned}
F\left(g_{0}, g_{1}, g_{2}, g_{3}, g_{4}\right)= & \nu_{4}\left(g_{0}, g_{1}, g_{2}, g_{3}, g_{4}\right) c_{(0124)}^{\dagger n_{3}(0124)} c_{(0234)}^{\dagger n_{3}(0234)} \\
& \times c_{(0123)}^{n_{3}(0123)} c_{(0134)}^{n_{3}(0134)} c_{(1234)}^{n_{3}(1234)} \\
& \times X\left[\tilde{n}_{2}\left(g_{i}, g_{j}, g_{k}\right)\right]
\end{aligned}
$$

Here, $c_{(0124)}^{\dagger n_{3}(0124)}$ is the abbreviation of $c_{(0124)}^{\dagger n_{3}\left(g_{0}, g_{1}, g_{2}, g_{4}\right)}$, which is the creation operator for $c$ fermions at tetrahedron $\langle 0124\rangle$. The $X\left[\tilde{n}_{2}\right]$ operator in the above $F$ move from the resolved dual lattice $\tilde{\mathcal{P}}$ to $\tilde{\mathcal{P}}^{\prime}$ has the following general expression:

$$
\begin{aligned}
X\left[\tilde{n}_{2}\left(g_{i}, g_{j}, g_{k}\right)\right]= & P_{\tilde{\mathcal{P}}} \cdot \gamma_{012 A}^{\tilde{n}_{2}^{2}\left(g_{0}, g_{1}, g_{2}, g_{3}, g_{4}\right)}, \\
P_{\tilde{\mathcal{P}}}= & \left(\prod_{\text {loop } i \text { in }\left(\tilde{\mathcal{P}}, \tilde{\mathcal{P}}^{\prime}\right)} 2^{\left(L_{i}-1\right) / 2}\right) \\
& \times\left(\prod_{\text {Majorana pairs }\langle a, b\rangle \text { in } \tilde{\mathcal{P}}} P_{a, b}\right) \\
& \times\left(\prod_{\text {link }\langle i j k\rangle \notin \tilde{\mathcal{P}}} P_{i j k A, i j k B}\right),
\end{aligned}
$$

where $2 L_{i}$ is the length of the $i$ th loop in the transition graph of dimer configurations in $\tilde{\mathcal{P}}$ and $\tilde{\mathcal{P}}^{\prime}$, and $P_{a, b}=$ $\left(1-i \gamma_{a} \gamma_{b}\right) / 2$ is the projection operator for Majorana pairs $\langle a, b\rangle$ (from vertex $a$ to vertex $b$ ). The projection operators in the second parentheses project the state in the right-hand figure to the Majorana dimer configuration states in the lefthand figure. The projection operators in the third parentheses are the vacuum projection operators for those Majorana fermions that do not appear in the left-hand figure explicitly [this is similar to the projection $P_{02 A, 02 B}$ in Eq. (25) of the 2D case]. An explicit expression of $X$ for the Majorana pair configuration in Eqs. (40) and (41) is

$$
\begin{aligned}
X\left[\tilde{n}_{2}\right]= & 2\left(P_{024 B, 234 B} P_{012 A, 024 A}\right) \\
& \times\left(P_{013 A, 013 B} P_{123 A, 123 B} P_{134 A, 134 B}\right) \gamma_{012 A}
\end{aligned}
$$

Note that the normalization factor in the front of a general $X$ operator is the same as Eq. (26). For the $F$ move in
Eqs. (40) and (41), there is only one loop in the Majorana dimer transition graph with length bigger than two: $012 A-024 A-024 B-234 B-123 A-123 B-012 A$. So the normalization factor is $2^{(6 / 2-1) / 2}=2$, as shown in Eq. (44).

When the $F$ move changes the Majorana fermion parity, the last term of the $X$ operator is the Majorana fermion operator $\gamma_{012 A}=a_{(012)}+a_{(012)}^{\dagger}$. The $X$ operator is now an operator with an odd number of $a$ fermion creation or annihilation operators, which changes the fermion parity of the state. We check that, for all possible Kitaev's Majorana chain configurations, the loop-breaking Kasteleyn orientation in the transition graph of the two Majorana dimer states always contains vertex $012 A$. Therefore, the $X$ operator with $\gamma_{012 A}$ should indeed project the state to the desired Majorana configuration state (not 0 ). In fact, $\gamma_{234 B}$ is also an allowed choice. We calculate the consistent equation of $\nu_{4}$ for both choices later.

The fermionic local unitary transformation $F$ should also be $G_{b}$ symmetric.

$$
F\left(g_{0}, g_{1}, g_{2}, g_{3}, g_{4}\right)=F\left(g g_{0}, g g_{1}, g g_{2}, g g_{3}, g g_{4}\right)
$$

for all $g \in G_{b}$ if $G_{b}$ is a unitary symmetry group. So, $\nu_{4}\left(g_{0}, g_{1}, g_{2}, g_{3}, g_{4}\right), n_{3}\left(g_{0}, g_{1}, g_{2}, g_{3}\right)$, and $\tilde{n}_{2}\left(g_{0}, g_{1}, g_{2}\right)$ are all cochains that are invariant under unitary $g$ action. [We note that $\nu_{4}\left(g g_{0}, g g_{1}, g g_{2}, g g_{3}, g g_{4}\right)=\nu_{4}^{*}\left(g_{0}, g_{1}, g_{2}, g_{3}, g_{4}\right)$ for antiunitary $g$ action.]

Similar to the $1 \mathrm{D}$ and $2 \mathrm{D}$ cases, we can use FSLU to redefine the basis state $\left|\left\{g_{n}\right\}\right\rangle$ as

$$
\begin{aligned}
\left|\left\{g_{n}\right\}\right\rangle^{\prime}= & U_{\mu_{3}, m_{2}}\left|\left\{g_{n}\right\}\right\rangle \\
= & \prod_{\langle i j k l\rangle} \mu_{3}\left(g_{i}, g_{j}, g_{k}, g_{l}\right)^{s_{i j k l\rangle}} \prod_{\langle i j k\rangle}\left[f_{i j k B}^{m_{2}\left(g_{i}, g_{j}, g_{k}\right)} f_{i j k A}^{m_{2}\left(g_{i}, g_{j}, g_{k}\right)}\right] \\
& \cdot \prod_{\langle i j k l\rangle}\left[f_{j k l A}^{\dagger m_{2}\left(g_{j}, g_{k}, g_{l}\right)} f_{i j l A}^{\dagger m_{2}\left(g_{i}, g_{j}, g_{l}\right)} f_{i k l B}^{\dagger m_{2}\left(g_{i}, g_{k}, g_{l}\right)} f_{i j k B}^{\dagger m_{2}\left(g_{i}, g_{j}, g_{k}\right)}\right] \\
& \times\left|\left\{g_{n}\right\}\right\rangle,
\end{aligned}
$$

where we first create four complex fermions $f_{j k l A}, f_{i j l A}$, $f_{i k l B}$, and $f_{i j k B}$ near the four triangles of the tetrahedron $\langle i j k l\rangle(i<j<k<l)$, and then annihilate the two fermions $f_{i j k A}$ and $f_{i j k B}$ on the two sides of triangle $\langle i j k\rangle$ when 
gluing the two tetrahedra sharing triangle $\langle i j k\rangle$. To preserve the fermion parity and be symmetric, $m_{2}$ should be a 2-cocycle (with $\mathbb{Z}_{2}$ coefficient): $m_{2}\left(g g_{i}, g g_{j}, g g_{k}\right)=$ $m_{2}\left(g_{i}, g_{j}, g_{k}\right)$ and $d m_{2}\left(g_{i}, g_{j}, g_{k}, g_{l}\right)=0 . \mu_{3}\left(g_{i}, g_{j}, g_{k}, g_{l}\right)$ is a phase factor associated with tetrahedron $\langle i j k l\rangle$, and $s_{\langle i j k l\rangle}= \pm 1$ denotes the orientation of the tetrahedron. After tedious calculation of eliminating all $f$ fermions in the new $F$ move operator $F^{\prime}=U_{\mu_{3}, m_{2}} F U_{\mu_{3}, m_{2}}^{\dagger}$, one finds that the phase factor in Eq. (42) becomes

$\nu_{4}^{\prime}\left(g_{0}, g_{1}, g_{2}, g_{3}, g_{4}\right)=\nu_{4}\left(g_{0}, g_{1}, g_{2}, g_{3}, g_{4}\right) \frac{\mu_{3}\left(g_{1}, g_{2}, g_{3}, g_{4}\right) \mu_{3}\left(g_{0}, g_{1}, g_{3}, g_{4}\right) \mu_{3}\left(g_{0}, g_{1}, g_{2}, g_{3}\right)}{\mu_{3}\left(g_{0}, g_{2}, g_{3}, g_{4}\right) \mu_{3}\left(g_{0}, g_{1}, g_{2}, g_{4}\right)}(-1)^{S q^{2}\left(m_{2}\right)\left(g_{0}, g_{1}, g_{2}, g_{3}, g_{4}\right)}$,

where $S q^{2}\left(m_{2}\right)\left(g_{0}, g_{1}, g_{2}, g_{3}, g_{4}\right)=\left(m_{2} \smile m_{2}\right)\left(g_{0}, g_{1}, g_{2}, g_{3}, g_{4}\right)=m_{2}\left(g_{0}, g_{1}, g_{2}\right) m_{2}\left(g_{2}, g_{3}, g_{4}\right)$ is the Steenrod square of $m_{2}$. If $\nu_{4}$ and $\nu_{4}^{\prime}$ can be related by the above equation, then they correspond to the same 3D FSPT phase. This is also consistent with the general result in Eq. (14) [42].

In addition to the standard (2-3) move, there are (2-0) moves as FSLU that can change the vertex number. One example of the Majorana fermion configuration for a (2-0) move is
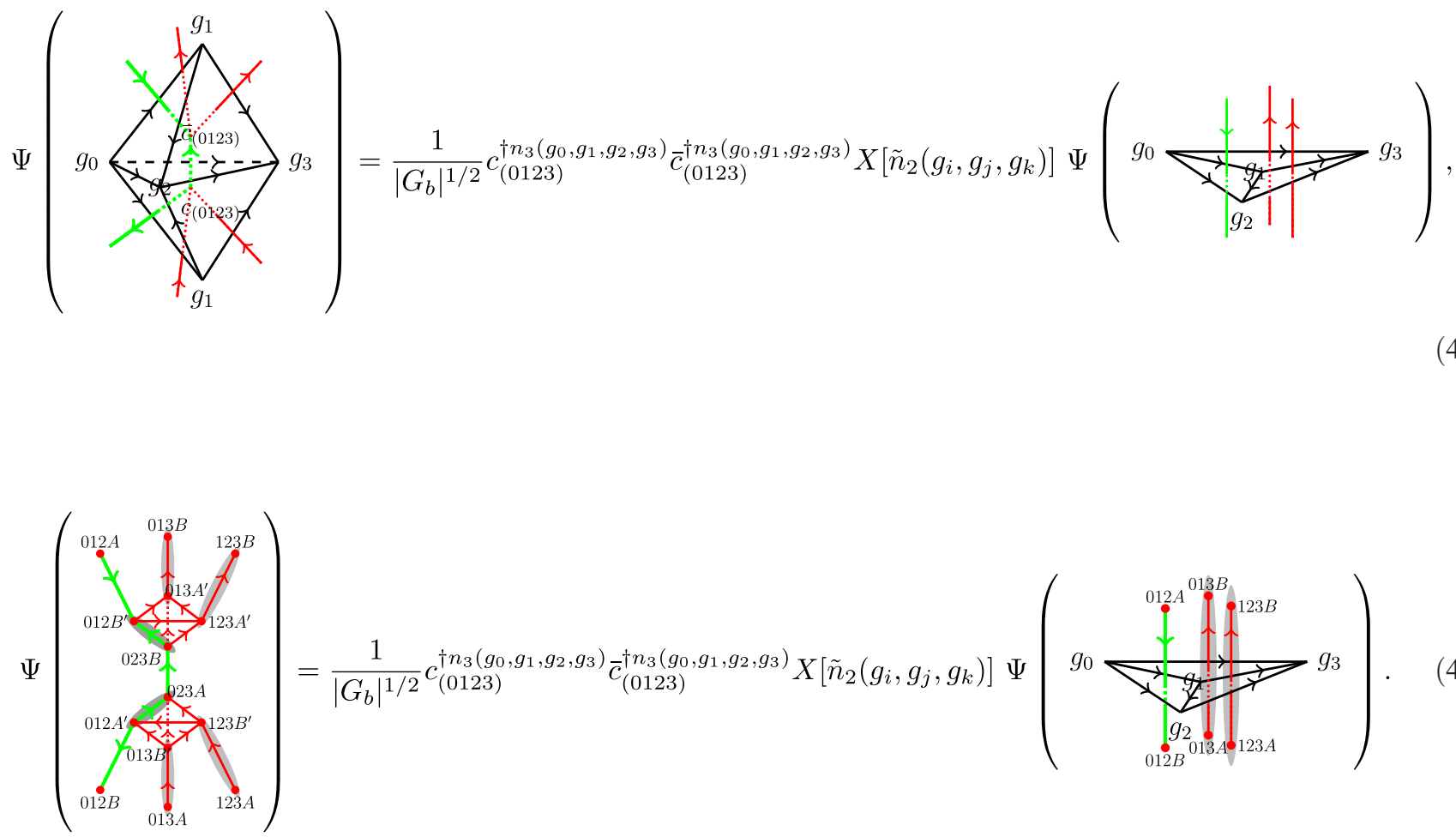

The notations and conventions of vacuum pairs are similar to those in the 2D case in the discussion following Eq. (30). $c_{(0123)}$ and $\bar{c}_{(0123)}$ fermions are at the centers of the lower and upper tetrahedra, respectively. An explicit expression for the $X$ operator is

$$
\begin{aligned}
X\left[\tilde{n}_{2}\right]= & 2^{3 / 2} P_{023 B, 012 B^{\prime}} P_{012 A^{\prime}, 023 A} P_{013 A^{\prime}, 013 B} P_{123 A^{\prime}, 123 B} \\
& \times P_{013 A, 013 B^{\prime}} P_{123 A, 123 B^{\prime}} .
\end{aligned}
$$

Note that the normalization factor in the front of the $X$ operator is $\left(2^{(4 / 2-1) / 2}\right)^{3}=2^{3 / 2}$, according to Eq. (26). This is because there are three loops in the Majorana dimer transition graph with length bigger than two:
012 $B^{\prime}-023 B-023 A-012 A^{\prime}-012 B^{\prime}, \quad 013 B-013 A^{\prime}-013 B^{\prime}-$ $013 A-013 B$, and $123 B-123 A^{\prime}-123 B^{\prime}-123 A-123 B$. Using two kinds of (2-0) moves (the other one is shown in Supplemental Material [72]) and the standard (2-3) move, we can deduce all (1-4) moves and other (2-3) moves from the standard $F$ move in Eqs. (40) and (41) (see Supplemental Material for all details [72]).

\section{Fermionic hexagon equations}

The consistent equation for the moves defined in the above section is the fermionic hexagon equation (see Fig. 20), which is a higher dimensional version of the fermionic pentagon equation for $(2+1) \mathrm{D}$ fermionic 


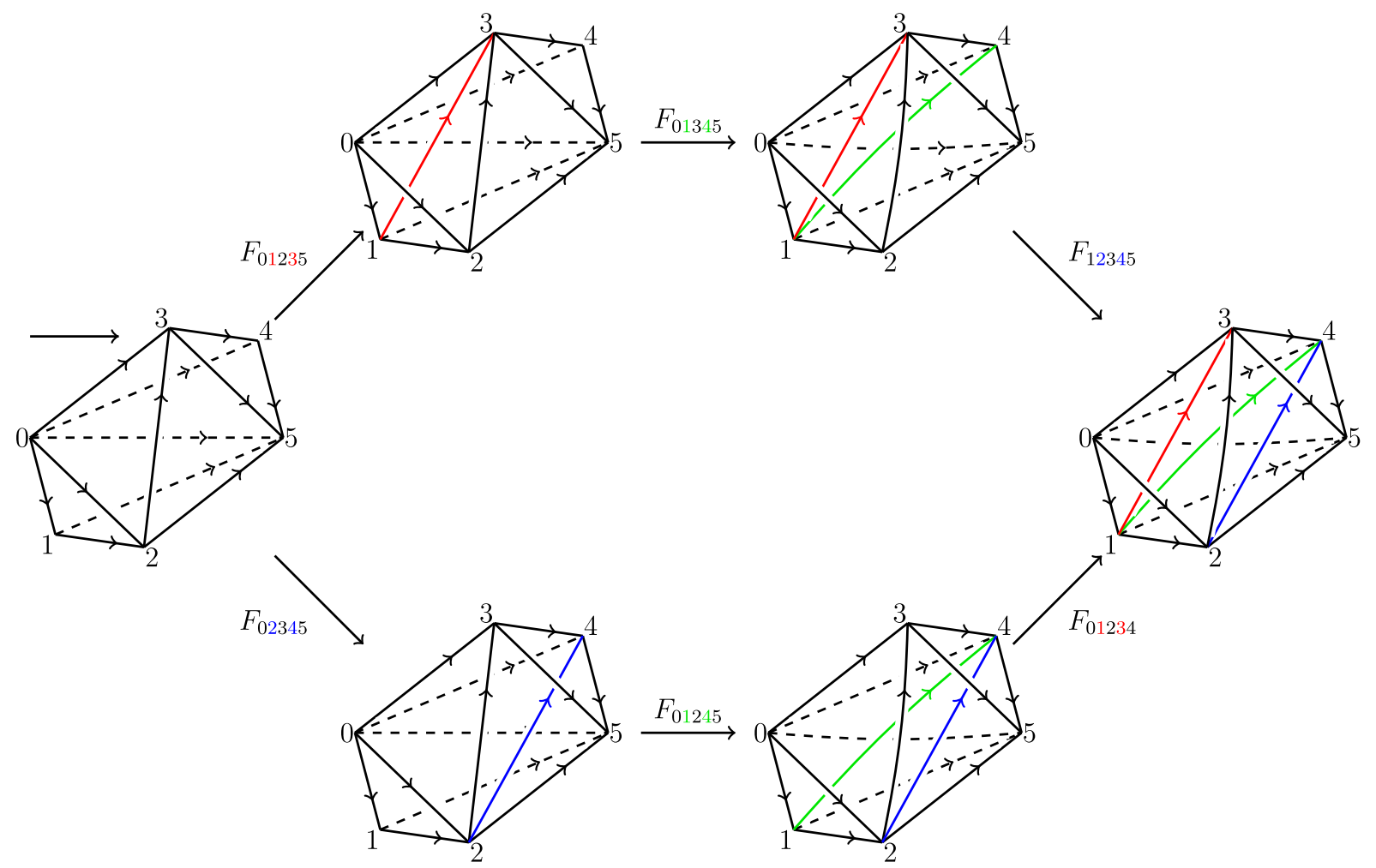

FIG. 20. Fermionic fusion hexagon equation. All of the $F$ moves are of the standard (2-3) move [see Eqs. (40) and (41)]. Colored numbers $i$ and $j$ in the subscript of $F$ indicate that the link $\langle i j\rangle$ with the same color is added after this $F$ move. All six $F$ moves do not introduce new singular lines and surfaces. There is a global direction from left to right such that the vertex with the smaller number has the earlier order.

topological phases [63]. Reordering the fermionic operators in the fermionic hexagon equation will twist the bosonic cocycle equation for $\nu_{4}$ :

$\left(d \nu_{4}\right)\left(g_{0}, g_{1}, g_{2}, g_{3}, g_{4}, g_{5}\right)=\mathcal{O}\left(g_{0}, g_{1}, g_{2}, g_{3}, g_{4}, g_{5}\right)$.

The phase twist or obstruction on the left-hand side has three terms,

$$
\mathcal{O}=\mathcal{O}_{c} \mathcal{O}_{c \gamma} \mathcal{O}_{\gamma},
$$

resulting from three different fermion phase factors: (1) $\mathcal{O}_{c}$ from reordering $c$ fermions, (2) $\mathcal{O}_{c \gamma}$ from reordering $c$ fermions and $\gamma$ Majorana fermions, and (3) $\mathcal{O}_{\gamma}$ from reordering $\gamma$ Majorana fermions. The final result of the phase twist is

$$
\begin{aligned}
\mathcal{O}(012345)= & (-1)^{\left(n_{3} \smile_{1} n_{3}\right)(012345)+\left(n_{3} \smile_{2} d n_{3}\right)(012345)+d n_{3}(02345) d n_{3}(01245) d n_{3}(01234)} \\
& \times i^{d n_{3}(01235) d n_{3}(02345)+d n_{3}(01345) d n_{3}(12345)} \times(-i)^{d n_{3}(02345) d n_{3}(01245)+d n_{3}(02345) d n_{3}(01234)} .
\end{aligned}
$$

Here, (012345) is the abbreviation of $\left(g_{0}, g_{1}, g_{2}, g_{3}, g_{4}, g_{5}\right)$. Note that the obstruction depends only on $n_{3}$ and $d n_{3}$ (or $\tilde{n}_{2}^{2}$ through the fermion parity equation $d n_{3}=\tilde{n}_{2}^{2}$ ). In the following, we derive the above obstruction equation in detail.

The first part $\mathcal{O}_{c}$ contains the special group supercohomology results [42], which are obtained by reordering $c$ fermion operators:

$$
\begin{aligned}
\mathcal{O}_{c}(012345)= & (-1)^{\left[S q^{2}\left(n_{3}\right)+d n_{3} \smile_{2} n_{3}\right](012345)}=(-1)^{\left[n_{3} \smile_{1} n_{3}+d n_{3} \smile_{2} n_{3}\right](012345)} \\
= & (-1)^{n_{3}(0345) n_{3}(0123)+n_{3}(0145) n_{3}(1234)+n_{3}(0125) n_{3}(2345)} \\
& \times(-1)^{d n_{3}(01234) n_{3}(0145)+d n_{3}(02345) n_{3}(0125)+d n_{3}(01234) n_{3}(1245)+d n_{3}(01345) n_{3}(1235)+d n_{3}(01234) n_{3}(2345)+d n_{3}(01245) n_{3}(2345)} .
\end{aligned}
$$


Here, $\smile_{i}$ is Steenrod's $i$ th cup product [75]. Apart from the term $S q^{2}\left(n_{3}\right)$, there is an additional term $d n_{3} \smile_{2} n_{3}$ because of $d n_{3}=\tilde{n}_{2}^{2} \neq 0$.

The second part $\mathcal{O}_{c \gamma}$ comes from reordering $c$ fermions and $\gamma$ Majorana fermions (we always put $c$ fermions in front of $\gamma$ fermions). For example, the composition of
$F_{01345}$ and $F_{01235}$ gives a sign $(-1)^{d n_{3}(01345) d n_{3}(01235)}$. In total, the upper path of three $F$ moves gives the power $d n_{3}(01345) d n_{3}(01235)+d n_{3}(12345) d n_{3}(01345)+$ $d n_{3}(12345) d n_{3}(01235)$, whereas the lower path gives $d n_{3}(01245) d n_{3}(01234)+d n_{3}(02345) d n_{3}(01234)+$ $d n_{3}(02345) d n_{3}(01245)$. The final result is then

$$
\begin{aligned}
& \mathcal{O}_{c \gamma}(012345) \\
& \quad=(-1)^{\left(d n_{3} \smile_{3} d n_{3}\right)\left(g_{0}, g_{1}, g_{2}, g_{3}, g_{4}, g_{5}\right)} \\
& \quad=(-1)^{d n_{3}(01245) d n_{3}(01234)+d n_{3}(01235) d n_{3}(01345)+d n_{3}(02345) d n_{3}(01234)+d n_{3}(02345) d n_{3}(01245)+d n_{3}(01235) d n_{3}(12345)+d n_{3}(01345) d n_{3}(12345)} .
\end{aligned}
$$

After adding coboundary $\quad(-1)^{d\left(n_{3} \smile_{3} d n_{3}\right)}=$ $(-1)^{d n_{3} \smile_{3} d n_{3}+n_{3} \smile_{2} d n_{3}+d n_{3} \smile_{2} n_{3}}$ to the combination of the phase factor $\mathcal{O}_{c}$ and $\mathcal{O}_{c \gamma}$, we obtain a simpler expression:

$$
\mathcal{O}_{c} \mathcal{O}_{c \gamma}=(-1)^{n_{3} \smile_{1} n_{3}+n_{3} \smile_{2} d n_{3}} .
$$

Note that adding the coboundary changes the $U(1)$-cochain $\nu_{4} \rightarrow(-1)^{n_{3} \smile_{3} d n_{3}} \nu_{4}$.

We now turn to the subtlest part $\mathcal{O}_{\gamma}$ coming from a decorated Majorana chain. In addition to $\pm 1, \mathcal{O}_{\gamma}$ can take values in $\pm i$ (see Supplemental Material for the physical origin of this purely imaginary phase factor [72]). If all six $F$ moves do not change the Majorana fermion parity, then $X_{\gamma}$ operators in $F$ moves are merely projections with an even number of $\gamma$ Majorana operators. The $c$ fermions and Majorana fermions are decoupled, and both $\mathcal{O}_{c \gamma}$ and $\mathcal{O}_{\gamma}$ are trivial. The obstruction $\mathcal{O}$ is the same as the special group supercohomology result. Therefore, we need to check only the case in which some of the six $F$ moves in Fig. 20 change the Majorana fermion parity; i.e., some of $\tilde{n}_{2}^{2}(01234), \quad \tilde{n}_{2}^{2}(01245), \quad \tilde{n}_{2}^{2}(02345), \quad \tilde{n}_{2}^{2}(01345)$, $\tilde{n}_{2}^{2}(01235)$, and $\tilde{n}_{2}^{2}(12345)$ are equal to one. We denote the six $X$ operators in $F$ moves as $X_{01235}=P_{1} \gamma_{012 A}^{\tilde{n}_{2}^{2}(01235)}$, $X_{01345}=P_{2} \gamma_{013 A}^{\tilde{n}_{2}^{2}(01345)}, \quad X_{12345}=P_{3} \gamma_{123 A}^{\tilde{n}_{2}^{2}(12345)}, \quad X_{02345}=$ $P_{1} \gamma_{023 A}^{\tilde{n}_{2}^{2}(02345)}, \quad X_{01245}=P_{4} \gamma_{012 A}^{\tilde{n}_{2}^{2}(01245)}$, and $X_{01234}=$ $P_{5} \gamma_{012 A}^{\tilde{n}_{2}^{2}(01234)}$. Here, $P_{i}$ means the projection operator onto the Majorana chain configuration of the $i$ th figure in the hexagon equation. $\mathcal{O}_{\gamma}$ is defined as the Majorana chain phase difference of the upper and lower paths in the hexagon equation

$$
\begin{aligned}
& \left.X_{02345} X_{01245} X_{01234} \mid \text { final }\right\rangle \\
& \left.\quad=\mathcal{O}_{\gamma}(012345) X_{01235} X_{01345} X_{12345} \mid \text { final }\right\rangle,
\end{aligned}
$$

where |final $\rangle$ is the state of the Majorana chain configuration in the rightmost figure of the hexagon equation Fig. 21. We can calculate $\mathcal{O}_{\gamma}$ from the expression

$$
\begin{aligned}
\mathcal{O}_{\gamma}(012345) & =\left\langle\text { final }\left|X_{12345}^{\dagger} X_{01345}^{\dagger} X_{01235}^{\dagger} X_{02345} X_{01245} X_{01234}\right| \text { final }\right\rangle \\
& =\left\langle\text { final }\left|\gamma_{123 A}^{\tilde{n}_{2}^{2}(12345)} P_{3} \gamma_{013 A}^{\tilde{n}_{2}^{2}(01345)} P_{2} \gamma_{012 A}^{\tilde{n}_{2}^{2}(01235)} P_{1} P_{1} \gamma_{023 A}^{\tilde{n}_{2}^{2}(02345)} P_{4} \gamma_{012 A}^{\tilde{n}_{2}^{2}(01245)} P_{5} \gamma_{012 A}^{\tilde{n}_{2}^{2}(01234)}\right| \text { final }\right\rangle .
\end{aligned}
$$

The above equation suggests that $\mathcal{O}_{\gamma}$ depends only on the values of $\tilde{n}_{2}^{2}(01235), \tilde{n}_{2}^{2}(01345), \tilde{n}_{2}^{2}(12345), \tilde{n}_{2}^{2}(02345)$, $\tilde{n}_{2}^{2}(01245)$, and $\tilde{n}_{2}^{2}(01234)$, i.e., the Majorana parity changes of the six $F$ moves.

Consider, for example, the case in which only $F_{01345}$ and $F_{12345}$ change the Majorana fermion parity $[(0,1,1,0,0,0)]$ in the eighth row of Table III. See Fig. 22 for an example of $\tilde{n}_{2}\left(g_{i}, g_{j}, g_{k}\right)$ satisfying this condition. We can expand projection operators $P_{i}$ to Majorana fermion operators. For simplicity, we can consider the term with only contributions $-i \gamma_{013 A} \gamma_{013 B}$ and $-i \gamma_{123 A} \gamma_{123 B}$ from $P_{1}$. We can also add $-i \gamma_{013 B} \gamma_{123 B}$, which equals 1 when acting on $\mid$ final $\rangle$ in front of this state. The result is then

$$
\begin{aligned}
\left.\mathcal{O}_{\gamma}(012345)\right|_{(0,1,1,0,0,0)} & =\left\langle\text { final }\left|\gamma_{123 A} P_{3} \gamma_{013 A} P_{2} P_{1} P_{4} P_{5} P_{6}\right| \text { final }\right\rangle \\
& =\left\langle\text { final }\left|\gamma_{123 A} \gamma_{013 A}\left(-i \gamma_{013 A} \gamma_{013 B}\right)\left(-i \gamma_{123 A} \gamma_{123 B}\right)\left(-i \gamma_{013 B} \gamma_{123 B}\right)\right| \text { final }\right\rangle \\
& =i
\end{aligned}
$$




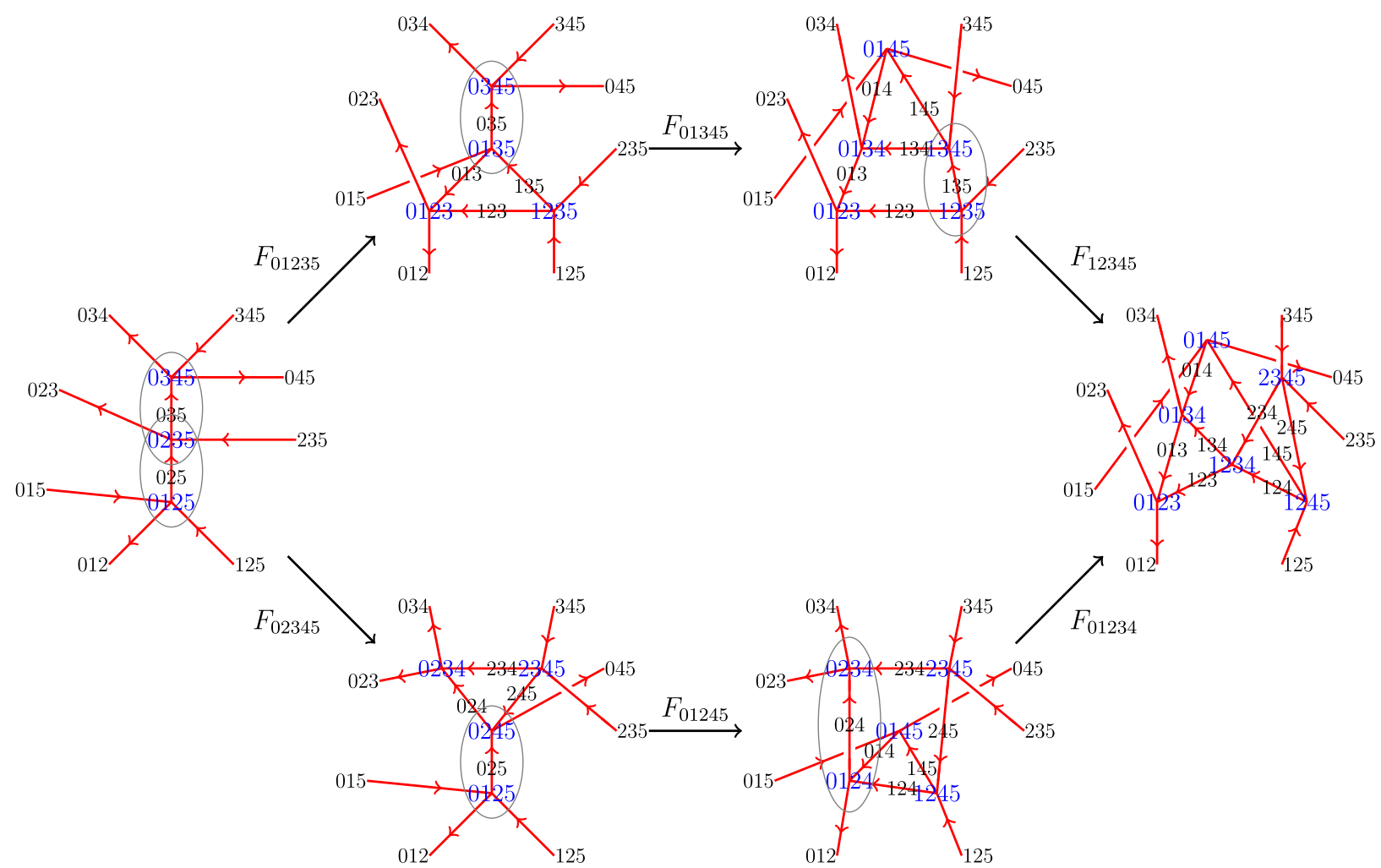

FIG. 21. Fermionic fusion hexagon equation on dual lattice $\mathcal{P}$.

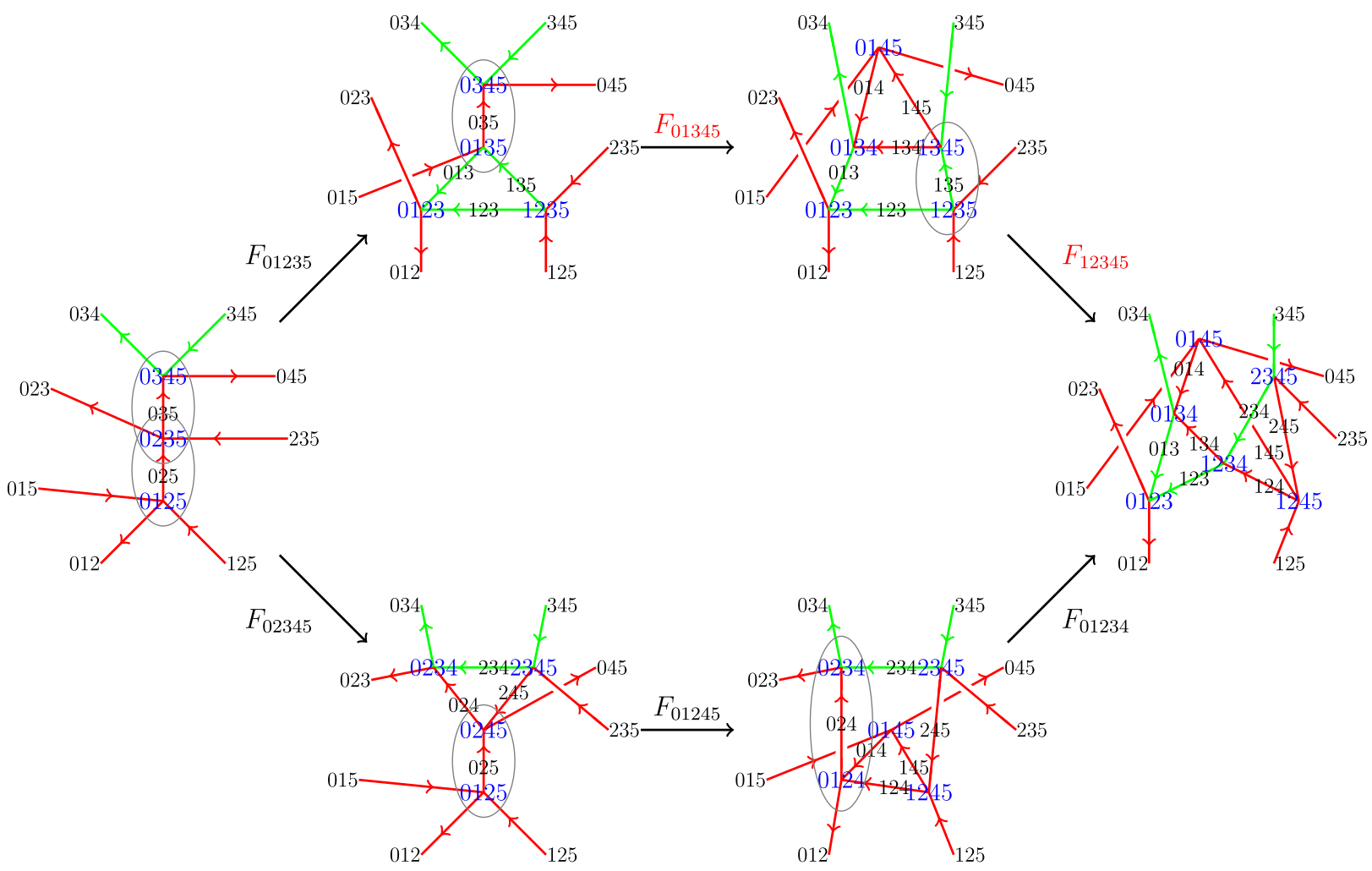

FIG. 22. One example of Kitaev's Majorana chain decoration configuration of fermionic fusion hexagon equation. Green lines indicate that these dual links are decorated by Kitaev's Majorana chains. Among the six $F$ moves for this configuration, only $F_{01345}$ and $F_{12345}$ (red color in the figure) change the Majorana fermion parity. Therefore, this choice of $\tilde{n}_{2}\left(g_{i}, g_{j}, g_{k}\right)$ belongs to the $(0,1,1,0,0,0)$ row in Table III and has obstruction $\mathcal{O}_{\gamma}=i$. 
We assume that there are only two Kitaev strings meeting at tetrahedron $\langle 0123\rangle$. [This is always true for all possible choices of $\tilde{n}_{2}\left(g_{i}, g_{j}, g_{k}\right)$ that belong to the eighth row of Table III.] Similarly, one can calculate other choices of $\tilde{n}_{2}$, and at last obtain the results listed in Table III. The final results can be summarized to the expression

$$
\begin{aligned}
\mathcal{O}_{\gamma}(012345)= & i^{\tilde{n}_{2}^{2}(01235) \tilde{n}_{2}^{2}(02345)+\tilde{n}_{2}^{2}(01345) \tilde{n}_{2}^{2}(12345)} \\
& \times(-i)^{\tilde{n}_{2}^{2}(02345) \tilde{n}_{2}^{2}(01245)+\tilde{n}_{2}^{2}(02345) \tilde{n}_{2}^{2}(01234)} \\
& \times(-1)^{\tilde{n}_{2}^{2}(02345) \tilde{n}_{2}^{2}(01245) \tilde{n}_{2}^{2}(01234)}
\end{aligned}
$$

$$
\begin{aligned}
\mathcal{O}_{\gamma}(012345)= & i^{\tilde{n}_{2}^{2}(01235) \tilde{n}_{2}^{2}(02345)+\tilde{n}_{2}^{2}(01245) \tilde{n}_{2}^{2}(01234)} \\
& \times(-i)^{\tilde{n}_{2}^{2}(01235) \tilde{n}_{2}^{2}(01345)+\tilde{n}_{2}^{2}(01235) \tilde{n}_{2}^{2}(12345)} \\
& \times(-1)^{\tilde{n}_{2}^{2}(01235) \tilde{n}_{2}^{2}(01345) \tilde{n}_{2}^{2}(12345)} .
\end{aligned}
$$

By checking all possible choices of $\tilde{n}_{2}$ numerically, we find that the above expression of $\mathcal{O}_{\gamma}$ equals exactly to

$$
\mathcal{O}_{\gamma}(012345)=(-i)^{\left[\tilde{n}_{2} \smile\left(\tilde{n}_{2} \smile_{1} \tilde{n}_{2}\right)\right](012345)}(-1)^{\tilde{n}_{2}^{2}(01235) \tilde{n}_{2}^{2}(02345)} .
$$

Combining this $\mathcal{O}_{\gamma}$ result with $\mathcal{O}_{c} \mathcal{O}_{c \gamma}$ in Eq. (56), we obtain the obstruction claimed in Eq. (53).

As discussed above, we can also use the convention $X_{01234}=P_{\mathcal{P}} \gamma_{234 B}^{\tilde{n}_{2}^{2}(01234)}$ in the definition of a standard $F$ move. The results using different conventions may differ from each other by some coboundaries. The obstruction from Majorana fermions in the convention $\gamma_{234 B}$ is
This can be obtained from the Pontrjagin dual of the fourdimensional spin bordism [76].

We note that although $\mathcal{O}$ takes values in $\mathbb{Z}_{4}$, the additivity property actually requires $\mathcal{O}$ to be a cohomology map on $\mathbb{Z}_{8}$; see Supplemental Material for details [72]. Thus, to find a solution for $\nu_{4}$, we must define an obstruction-free subgroup of $H^{2}\left(G_{b}, \mathbb{Z}_{2}\right)$, which is formed

TABLE III. Calculations of $\mathcal{O}_{\gamma}$ from all possible Kitaev chain configurations in the hexagon equation. The first column has entries

\begin{tabular}{|c|c|c|c|c|c|}
\hline Fermion parity changes & $\gamma$ operators in Eq. $(58)$ & Line $(2$ strings at $\langle 0123\rangle)$ & Line (4 strings at $\langle 0123\rangle$ ) & $\left.\mathcal{O}_{\gamma}\right|_{012 A}$ & $\left.\mathcal{O}_{\gamma}\right|_{234 B}$ \\
\hline$(0,0,0,0,0,0)$ & $\cdots$ & $\ldots$ & $\cdots$ & 1 & 1 \\
\hline$(1,1,0,0,0,0)$ & $\gamma_{012 A}, \gamma_{013 A}$ & $012 A-013 B-013 A$ & $\varnothing$ & 1 & $-i$ \\
\hline$(1,0,1,0,0,0)$ & $\gamma_{012 A}, \gamma_{123 A}$ & $012 A-123 B-123 A$ & $\varnothing$ & 1 & $-i$ \\
\hline$(1,0,0,1,0,0)$ & $\gamma_{012 A}, \gamma_{023 A}$ & 012A-023A & $\varnothing$ & $i$ & $i$ \\
\hline$(1,0,0,0,1,0)$ & $\gamma_{012 A}^{2}=1$ & $\ldots$ & $\cdots$ & 1 & 1 \\
\hline$(1,0,0,0,0,1)$ & $\gamma_{012 A}^{2}=1$ & $\cdots$ & $\cdots$ & 1 & 1 \\
\hline$(0,1,1,0,0,0)$ & $\gamma_{013 A}, \gamma_{123 A}$ & $013 A-013 B-123 B-123 A$ & $\varnothing$ & $i$ & 1 \\
\hline$(0,1,0,1,0,0)$ & $\gamma_{013 A}, \gamma_{023 A}$ & 013A-013B-023A & $\varnothing$ & 1 & 1 \\
\hline$(0,1,0,0,1,0)$ & $\gamma_{013 A}, \gamma_{012 A}$ & $013 A-013 B-012 A$ & $\varnothing$ & 1 & 1 \\
\hline$(0,1,0,0,0,1)$ & $\gamma_{013 A}, \gamma_{012 A}$ & $013 A-013 B-012 A$ & $\varnothing$ & 1 & 1 \\
\hline$(0,0,1,1,0,0)$ & $\gamma_{123 A}, \gamma_{023 A}$ & $123 A-123 B-023 A$ & $\varnothing$ & 1 & 1 \\
\hline$(0,0,1,0,1,0)$ & $\gamma_{123 A}, \gamma_{012 A}$ & $123 A-123 B-012 A$ & $\varnothing$ & 1 & 1 \\
\hline$(0,0,1,0,0,1)$ & $\gamma_{123 A}, \gamma_{012 A}$ & $123 A-123 B-012 A$ & $\varnothing$ & 1 & 1 \\
\hline$(0,0,0,1,1,0)$ & $\gamma_{023 A}, \gamma_{012 A}$ & $023 A-012 A$ & $\varnothing$ & $-i$ & 1 \\
\hline$(0,0,0,1,0,1)$ & $\gamma_{023 A}, \gamma_{012 A}$ & $023 A-012 A$ & $\varnothing$ & $-i$ & 1 \\
\hline$(0,0,0,0,1,1)$ & $\gamma_{012 A}^{2}=1$ & $\cdots$ & $\cdots$ & 1 & $i$ \\
\hline$(1,1,0,0,1,1)$ & $\gamma_{013 A}, \gamma_{012 A}^{3}$ & $012 A-013 B-013 A, 012 A$ & $\varnothing$ & 1 & 1 \\
\hline$(1,0,1,0,1,1)$ & $\gamma_{123 A}, \gamma_{012 A}^{3}$ & $012 A-123 B-123 A, 012 A$ & $\varnothing$ & 1 & 1 \\
\hline$(1,0,0,1,1,1)$ & $\gamma_{023 A}, \gamma_{012 A}^{3}$ & $012 A-023 A, 012 A$ & $\varnothing$ & $i$ & -1 \\
\hline$(1,1,1,1,0,0)$ & $\gamma_{012 A}, \gamma_{013 A}, \gamma_{123 A}, \gamma_{023 A}$ & $\varnothing$ & $\left\{\begin{array}{l}123 A-123 B-013 B-013 A \\
023 A-012 A\end{array}\right.$ & -1 & $i$ \\
\hline$(0,1,1,1,1,0)$ & $\gamma_{013 A}, \gamma_{123 A}, \gamma_{023 A}, \gamma_{012 A}$ & $\varnothing$ & $\left\{\begin{array}{l}123 A-123 B-013 B-013 A \\
023 A-012 A\end{array}\right.$ & 1 & 1 \\
\hline$(0,1,1,1,0,1)$ & $\gamma_{013 A}, \gamma_{123 A}, \gamma_{023 A}, \gamma_{012 A}$ & $\varnothing$ & $\left\{\begin{array}{l}123 A-123 B-013 B-013 \\
A 023 A-012 A\end{array}\right.$ & 1 & 1 \\
\hline$(1,1,1,1,1,1)$ & $\gamma_{013 A}, \gamma_{123 A}, \gamma_{023 A}, \gamma_{012 A}^{3}$ & $\varnothing$ & $\left\{\begin{array}{l}123 A-123 B-013 B-013 A \\
023 A-012 A\end{array}\right.$ & -1 & -1 \\
\hline
\end{tabular}
$\left[\tilde{n}_{2}^{2}(01235), \tilde{n}_{2}^{2}(01345), \tilde{n}_{2}^{2}(12345), \tilde{n}_{2}^{2}(02345), \tilde{n}_{2}^{2}(01245), \tilde{n}_{2}^{2}(01234)\right]$, indicating whether six $F$ moves in the hexagon equation change the Majorana fermion parity. The second column shows the $\gamma$ operators appearing in Eq. (58). The third and fourth columns are lines of Majorana dimer pairs $\left[\varnothing\right.$ means there are no $\tilde{n}_{2}\left(g_{i}, g_{j}, g_{k}\right)$ that have two or four strings at tetrahedron $\left.\langle 0123\rangle\right]$. The last two columns are the values of $\mathcal{O}_{\gamma}$ for $\tilde{n}_{2}\left(g_{i}, g_{j}, g_{k}\right)$ belonging to this row [the last column but one uses the convention $\gamma_{012 A}$ in Eq. (43), and the last column uses the convention $\gamma_{234 B}$ ]. Nontrivial results are labeled in red or blue. The results in this table can be summarized to the expression shown in Eq. (60) for convention $\gamma_{012 A}$ and in Eq. (61) for convention $\gamma_{234 B}$. 
by elements $\tilde{n}_{2} \in H^{2}\left(G_{b}, \mathbb{Z}_{2}\right)$ that simultaneously satisfy $S q^{2}\left(\tilde{n}_{2}\right)=0$ in $H^{4}\left(G_{b}, \mathbb{Z}_{2}\right)$ and $\mathcal{O}\left(\tilde{n}_{2}\right)=0$ in $H^{5}\left(G_{b}, U_{T}(1)\right)$, where $\mathcal{O}$ is some unknown cohomology operation (to the best of our knowledge) that maps $\tilde{n}_{2}$ satisfying $S q^{2}\left(\tilde{n}_{2}\right)=0$ in $H^{2}\left(G_{b}, \mathbb{Z}_{2}\right)$ into an element in $H^{5}\left(G_{b}, \mathbb{Z}_{8}\right) \subset H^{5}\left[G_{b}, U_{T}(1)\right]$. We note that $n_{3}$ is completely determined by $\tilde{n}_{2}$ and that any solution of $d n_{3}=\tilde{n}_{2}^{2}$ can be used in the above definition. Together with the special group supercohomolgy results, we conclude that the precise mathematical objects that classify 3D FSPT phases with a total symmetry $G_{f}=G_{b} \times \mathbb{Z}_{2}^{f}$ can also be summarized as three group cohomologies of the symmetry group $G_{b}, \tilde{B} H^{2}\left(G_{b}, \mathbb{Z}_{2}\right), B H^{3}\left(G_{b}, \mathbb{Z}_{2}\right)$, and $H_{\text {rigid }}^{4}\left(G_{b}, U_{T}(1)\right)$. Commuting projector parent Hamiltonians for all of these FSPT states can also be constructed on arbitrary 3D triangulations with a branching structure.

Finally, let us provide some physical arguments to support our classification scheme for 3D FSPT phases with a total symmetry $G_{f}=G_{b} \times \mathbb{Z}_{2}^{f}$ when $G_{b}$ is a unitary symmetry group. (a) From the concept of equivalence classes of FSLU transformations, if two fixed-point wave functions corresponded to the same phase, there would be a finite depth FSLU circuit connecting the two. Thus, it is obvious that the fixedpoint wave functions have the same algebraic data, e.g., cocycle solutions (up to coboundary transformation) from the above three group cohomologies of the symmetry group $G_{b}$, since these algebraic data depend only on "long distance" physics which cannot be changed by a finite depth FSLU circuit. In fact, a finite depth FSLU circuit can at most generate some coboundary transformations, e.g., the transformation defined in Eq. (47). (b) Our constructions are consistent with the spin-cobordism classifications for 3D FSPT phases. (c) Similar to the bosonic 3D SPT states, if we turn on background gauge field $G_{b}$ and couple it to the 3D FSPT states constructed here, the corresponding $G_{b}$ flux lines will carry new types of three-loop braiding statistics. Some examples from the $B H^{3}\left(G_{b}, \mathbb{Z}_{2}\right)$ layer are studied in a recent work [77]. We believe that nontrivial solutions from the layer $\tilde{B} H^{2}\left(G_{b}, \mathbb{Z}_{2}\right)$ will give rise to non-Abelian three-loop braiding statistics, and full details will be studied in our future work.

\section{DISCUSSION AND CONCLUSIONS}

We construct fixed-point wave functions for FSPT phases in two and three dimensions based on the novel concept of FSLU transformations. All of these FSPT states admit parent Hamiltonians consisting of commuting projectors on arbitrary triangulations with a branching structure. We believe that our construction will give rise to a complete classification for FSPT states with total symmetry $G_{f}=G_{b} \times \mathbb{Z}_{2}^{f}$ when $G_{b}$ is a unitary symmetry group. Mathematically, our constructions naturally define a general group supercohomology theory that generalizes the so-called special group supercohomology theory proposed in Ref. [42].
In particular, one can start with a spin manifold in an arbitrary spacial dimension $d_{\mathrm{sp}}$ and define the corresponding discrete spin structure via the Poincaré dual. Then, one can decorate Kitaev's Majorana chain onto the intersection lines of the $G_{b}$ symmetry domain walls if the first obstruction vanishes for elements $\tilde{n}_{d_{\mathrm{sp}}-1} \in H^{d_{\mathrm{sp}}-1}\left(G_{b}, \mathbb{Z}_{2}\right)$. That is, $S q^{2}\left(\tilde{n}_{d_{\mathrm{sp}}+1}\right)=0$ in $H^{d_{\mathrm{sp}}+1}\left(G_{b}, \mathbb{Z}_{2}\right)$, and the obstruction-free elements $\tilde{n}_{d_{\mathrm{sp}}-1} \in H^{d_{\mathrm{sp}}-1}\left(G_{b}, \mathbb{Z}_{2}\right)$ will give rise to all inequivalent patterns of Majorana chain decoration. Finally, by applying the wave function renormalization on arbitrary triangulations, one may derive twisted cocycle equations where the twisted factors define some unknown cohomologoy map $\mathcal{O}$ that maps elements in $H^{d_{\mathrm{sp}}-1}\left(G_{b}, \mathbb{Z}_{2}\right)$ satisfying $S q^{2}\left(\tilde{n}_{d_{\mathrm{sp}}-1}\right)=0$ in $H^{d_{\mathrm{sp}}+1}\left(G_{b}, \mathbb{Z}_{2}\right)$ into elements in $H^{d_{\mathrm{sp}}+2}\left(G_{b}, \mathbb{Z}_{8}\right) \subset H^{d_{\mathrm{sp}}+2}\left[G_{b}, U_{T}(1)\right]$, and the second obstruction-free condition requires $\mathcal{O}\left(\tilde{n}_{d_{\mathrm{sp}}-1}\right)=0$ in $H^{d_{\mathrm{sp}}+2}\left(G_{b}, U_{T}(1)\right)$. Elements $\tilde{n}_{d_{\mathrm{sp}}-1} \in H^{d_{\mathrm{sp}}-1}\left(G_{b}, \mathbb{Z}_{2}\right)$ satisfying both the first and second obstruction-free conditions may define a subgroup $\tilde{B} H^{d_{\mathrm{sp}}-1}\left(G_{b}, \mathbb{Z}_{2}\right) \in H^{d_{\mathrm{sp}}-1}\left(G_{b}, \mathbb{Z}_{2}\right)$, which allows us to write down another short exact sequence $0 \rightarrow \mathcal{H}^{d_{\mathrm{sp}}+1}\left[G_{f}, U_{T}(1)\right] \rightarrow H_{f}^{d_{\mathrm{sp}}+1}\left[G_{f}, U_{T}(1)\right] \rightarrow$ $\tilde{B} H^{d_{\mathrm{sp}}-1}\left(G_{b}, \mathbb{Z}_{2}\right) \rightarrow 0$ to define a general group supercohomology theory. We note that here $\mathcal{H}^{d_{\mathrm{sp}}+1}\left[G_{f}, U_{T}(1)\right]$ is the special group supercohomology class defined by Ref. [42].

In the future, it would be of great importance to understand the physical properties of 3D FSPT phases classified by general group supercohomology theory, e.g., understanding the braiding statistics of $G_{b}$-flux lines. Of course, constructing time-reversal symmetry-protected topological states with both $T^{2}=1$ and $T^{2}=P^{f}$ (where $P^{f}$ is the total fermion parity) is another interesting direction. It should also be interesting to investigate the phase transition theory among FSPT phases in arbitrary dimensions.

\section{ACKNOWLEDGMENTS}

We thank Zheng-Xin Liu and Jianwei Li for helpful discussions on solving the system of linear Diophantine equations. We also thank Shuo Yang for helping to calculate the equations numerically. Z.-C. G. acknowledges start-up support via Direct Grants No. 4053163 and No. 3132745 from The Chinese University of Hong Kong and funding from Hong Kong's Research Grants Council (ECS No. 2191110, GRF No. 14306714). We also would like to thank Tianhe-1A platform at the National Supercomputer Center in Tianjin and Tianhe-2 platform at the National Supercomputer Center in Guangzhou for computational support.

Note added.-Recently, we noticed a relevant work [78] considering similar problems. However, our expression for $\mathcal{O}_{\gamma}$ is slightly different from Ref. [78]. 
[1] Z.-C. Gu and X.-G. Wen, Tensor-Entanglement-Filtering Renormalization Approach and Symmetry-Protected Topological Order, Phys. Rev. B 80, 155131 (2009).

[2] X. Chen, Z.-C. Gu, Z.-X. Liu, and X. -G. Wen, SymmetryProtected Topological Orders in Interacting Bosonic Systems, Science 338, 1604 (2012).

[3] X. Chen, Z.-C. Gu, Z.-X. Liu, and X.-G. Wen, Symmetry Protected Topological Orders and the Group Cohomology of Their Symmetry Group, Phys. Rev. B 87, 155114 (2013).

[4] M.Z. Hasan and C.L. Kane, Colloquium: Topological Insulators, Rev. Mod. Phys. 82, 3045 (2010).

[5] X.-L. Qi and S.-C. Zhang, Topological Insulators and Superconductors, Rev. Mod. Phys. 83, 1057 (2011).

[6] E. Witten, Fermion Path Integrals and Topological Phases, Rev. Mod. Phys. 88, 035001 (2016).

[7] F. D. M. Haldane, Nonlinear Field Theory of Large-Spin Heisenberg Antiferromagnets: Semiclassically Quantized Solitons of the One-Dimensional Easy-Axis Néel State, Phys. Rev. Lett. 50, 1153 (1983).

[8] X.-G. Wen, Construction of Bosonic Symmetry-ProtectedTrivial States and Their Topological Invariants via $G \times S O(\infty)$ Nonlinear $\sigma$ Models, Phys. Rev. B 91, 205101 (2015).

[9] A. Kapustin, Symmetry Protected Topological Phases, Anomalies, and Cobordisms: Beyond Group Cohomology, arXiv:1403.1467.

[10] M. Levin and Z.-C. Gu, Braiding Statistics Approach to Symmetry-Protected Topological Phases, Phys. Rev. B 86, 115109 (2012).

[11] M. Cheng and Z.-C. Gu, Topological Response Theory of Abelian Symmetry-Protected Topological Phases in Two Dimensions, Phys. Rev. Lett. 112, 141602 (2014).

[12] C. Wang, C.-H. Lin, and Z.-C. Gu, Interacting Fermionic Symmetry-Protected Topological Phases in Two Dimensions, Phys. Rev. B 95, 195147 (2017).

[13] C. Wang and M. Levin, Braiding Statistics of Loop Excitations in Three Dimensions, Phys. Rev. Lett. 113, 080403 (2014).

[14] S. Jiang, A. Mesaros, and Y. Ran, Generalized Modular Transformations in $(3+1) \mathrm{D}$ Topologically Ordered Phases and Triple Linking Invariant of Loop Braiding, Phys. Rev. X 4, 031048 (2014).

[15] C. Wang and M. Levin, Topological Invariants for Gauge Theories and Symmetry-Protected Topological Phases, Phys. Rev. B 91, 165119 (2015).

[16] J. C. Wang and X.-G. Wen, Non-Abelian String and Particle Braiding in Topological Order: Modular SL $(3, \mathbb{Z})$ Representation and $(3+1)$-Dimensional Twisted Gauge Theory, Phys. Rev. B 91, 035134 (2015).

[17] C.-H. Lin and M. Levin, Loop Braiding Statistics in Exactly Soluble Three-Dimensional Lattice Models, Phys. Rev. B 92, 035115 (2015).

[18] A. Vishwanath and T. Senthil, Physics of ThreeDimensional Bosonic Topological Insulators: SurfaceDeconfined Criticality and Quantized Magnetoelectric Effect, Phys. Rev. X 3, 011016 (2013).

[19] C. Wang and T. Senthil, Boson Topological Insulators: A Window into Highly Entangled Quantum Phases, Phys. Rev. B 87, 235122 (2013).
[20] X. Chen, F. J. Burnell, A. Vishwanath, and L. Fidkowski, Anomalous Symmetry Fractionalization and Surface Topological Order, Phys. Rev. X 5, 041013 (2015).

[21] C. Wang, C.-H. Lin, and M. Levin, Bulk-Boundary Correspondence for Three-Dimensional Symmetry-Protected Topological Phases, Phys. Rev. X 6, 021015 (2016).

[22] P. Bonderson, C. Nayak, and X.-L. Qi, A Time-Reversal Invariant Topological Phase at the Surface of a $3 D$ Topological Insulator, J. Stat. Mech. (2013) P09016.

[23] C. Wang, A. C. Potter, and T. Senthil, Gapped Symmetry Preserving Surface State for the Electron Topological Insulator, Phys. Rev. B 88, 115137 (2013).

[24] L. Fidkowski, X. Chen, and A. Vishwanath, Non-Abelian Topological Order on the Surface of a 3D Topological Superconductor from an Exactly Solved Model, Phys. Rev. X 3, 041016 (2013).

[25] X. Chen, L. Fidkowski, and A. Vishwanath, Symmetry Enforced Non-Abelian Topological Order at the Surface of a Topological Insulator, Phys. Rev. B 89, 165132 (2014).

[26] Chong Wang and T. Senthil, Interacting Fermionic Topological Insulators/Superconductors in Three Dimensions, Phys. Rev. B 89, 195124 (2014).

[27] M. A. Metlitski, C. L. Kane, and M. P. A. Fisher, SymmetryRespecting Topologically Ordered Surface Phase of ThreeDimensional Electron Topological Insulators, Phys. Rev. B 92, 125111 (2015).

[28] X. Chen, Z.-C. Gu, and X.-G. Wen, Local Unitary Transformation, Long-Range Quantum Entanglement, Wave Function Renormalization, and Topological Order, Phys. Rev. B 82, 155138 (2010).

[29] K. Walker and Z. Wang, $(3+1)$-TQFTs and Topological Insulators, Front. Phys. 7, 150 (2012).

[30] F. J. Burnell, X. Chen, L. Fidkowski, and A. Vishwanath, Exactly Soluble Model of a Three-Dimensional SymmetryProtected Topological Phase of Bosons with Surface Topological Order, Phys. Rev. B 90, 245122 (2014).

[31] A. P. Schnyder, S. Ryu, A. Furusaki, and A. W. W. Ludwig, Classification of Topological Insulators and Superconductors in Three Spatial Dimensions, Phys. Rev. B 78, 195125 (2008).

[32] A. Kitaev, Periodic Table for Topological Insulators and Superconductors, AIP Conf. Proc. 1134, 22 (2009).

[33] L. Fidkowski and A. Kitaev, Effects of Interactions on the Topological Classification of Free Fermion Systems, Phys. Rev. B 81, 134509 (2010).

[34] X.-L. Qi, A New Class of (2+1)-Dimensional Topological Superconductors with $\mathbb{Z}_{8}$ Topological Classification, New J. Phys. 15, 065002 (2013).

[35] H. Yao and S. Ryu, Interaction Effect on Topological Classification of Superconductors in Two Dimensions, Phys. Rev. B 88, 064507 (2013).

[36] S. Ryu and S.-C. Zhang, Interacting Topological Phases and Modular Invariance, Phys. Rev. B 85, 245132 (2012).

[37] Z.-C. Gu and M. Levin, Effect of Interactions on TwoDimensional Fermionic Symmetry-Protected Topological Phases with $Z_{2}$ Symmetry, Phys. Rev. B 89, 201113 (2014).

[38] Y.-Z. You and C. Xu, Symmetry-Protected Topological States of Interacting Fermions and Bosons, Phys. Rev. B 90, 245120 (2014). 
[39] T. Morimoto, A. Furusaki, and C. Mudry, Breakdown of the Topological Classification $\mathbb{Z}$ for Gapped Phases of Noninteracting Fermions by Quartic Interactions, Phys. Rev. B 92, 125104 (2015).

[40] T. Neupert, C. Chamon, C. Mudry, and R. Thomale, Wire Deconstructionism of Two-Dimensional Topological Phases, Phys. Rev. B 90, 205101 (2014).

[41] C. Wang, A. C. Potter, and T. Senthil, Classification of Interacting Electronic Topological Insulators in Three Dimensions, Science 343, 6171 (2014).

[42] Z.-C. Gu and X.-G. Wen, Symmetry-Protected Topological Orders for Interacting Fermions: Fermionic Topological Nonlinear $\sigma$ Models and a Special Group Supercohomology Theory, Phys. Rev. B 90, 115141 (2014).

[43] X. Chen, Z.-C. Gu, and X.-G. Wen, Classification of Gapped Symmetric Phases in One-Dimensional Spin Systems, Phys. Rev. B 83, 035107 (2011).

[44] L. Fidkowski and A. Kitaev, Topological Phases of Fermions in One Dimension, Phys. Rev. B 83, 075103 (2011).

[45] A. Kapustin, R. Thorngren, A. Turzillo, and Z. Wang, Fermionic Symmetry Protected Topological Phases and Cobordisms, J. High Energy Phys. 12 (2015) 052.

[46] D. S. Freed, Short-Range Entanglement and Invertible Field Theories, arXiv:1406.7278.

[47] D. Gaiotto and A. Kapustin, Spin TQFTs and Fermionic Phases of Matter, Int. J. Mod. Phys. A 31, 1645044 (2016).

[48] L. Bhardwaj, D. Gaiotto, and A. Kapustin, State Sum Constructions of Spin-TFTs and String Net Constructions of Fermionic Phases of Matter, J. High Energy Phys. 04 (2017) 096.

[49] M. Barkeshli, P. Bonderson, M. Cheng, and Z. Wang, Symmetry, Defects, and Gauging of Topological Phases, arXiv: 1410.4540.

[50] M. Cheng, Z. Bi, Y.-Z. You, and Z.-C. Gu, Towards a Complete Classification of Symmetry-Protected Phases for Interacting Fermions in Two Dimensions, arXiv: 1501.01313.

[51] C. Wang, Braiding Statistics and Classification of TwoDimensional Charge-2m Superconductors, Phys. Rev. B 94, 085130 (2016).

[52] T. Lan, L. Kong, and X.-G. Wen, Classification of $(2+1) \mathrm{D}$ Topological Orders and SPT Orders for Bosonic and Fermionic Systems with On-Site Symmetries, Phys. Rev. B 95, 235140 (2017).

[53] D. S. Freed and M. J. Hopkins, Reflection Positivity and Invertible Topological Phases, arXiv:1604.06527.

[54] G. Brumfiel and J. Morgan, The Pontrjagin Dual of 3-Dimensional Spin Bordism, arXiv:1612.02860.

[55] A. Y. Kitaev, Unpaired Majorana Fermions in Quantum Wires, Phys. Usp. 44, 131 (2001).

[56] The fermion parity symmetry is gauged in such constructions.

[57] M. A. Metlitski, L. Fidkowski, X. Chen, and A. Vishwanath, Interaction Effects on 3D Topological Superconductors: Surface Topological Order from Vortex Condensation, the 16 Fold Way and Fermionic Kramers Doublets, arXiv: 1406.3032.

[58] A. Kitaev, http://www.ipam.ucla.edu/abstract/?tid=12389.
[59] R. Queiroz, E. Khalaf, and A. Stern, Classification of Interacting Fermionic Phases by Dimensional Reduction, Phys. Rev. Lett. 117, 206405 (2016).

[60] P. Putrov, J. Wang, and S.-T. Yau, Braiding Statistics and Link Invariants of Bosonic/Fermionic Topological Quantum Matter in $2+1$ and $3+1$ dimensions, Ann. Phys. (Amsterdam) 384, 254 (2017).

[61] M. Cheng, Z.-C. Gu, S. Jiang, and Y. Qi, Exactly Solvable Models for Symmetry-Enriched Topological Phases, Phys. Rev. B 96, 115107 (2017).

[62] C. Heinrich, F. Burnell, L. Fidkowski, and M. Levin, Symmetry Enriched String-Nets: Exactly Solvable Models for SET Phases, Phys. Rev. B 94, 235136 (2016).

[63] Z.-C. Gu, Z. Wang, and X.-G. Wen, Classification of TwoDimensional Fermionic and Bosonic Topological Orders, Phys. Rev. B 91, 125149 (2015).

[64] P. W. Kasteleyn, Dimer Statistics and Phase Transitions, J. Math. Phys. (N.Y.) 4, 287 (1963).

[65] D. Cimasoni and N. Reshetikhin, Dimers on Surface Graphs and Spin Structures. I, Commun. Math. Phys. 275, 187 (2007).

[66] D. Cimasoni and N. Reshetikhin, Dimers on Surface Graphs and Spin Structures. II, Commun. Math. Phys. 281, 445 (2008).

[67] N. Tarantino and L. Fidkowski, Discrete Spin Structures and Commuting Projector Models for Two-Dimensional Fermionic Symmetry-Protected Topological Phases, Phys. Rev. B 94, 115115 (2016).

[68] B. Ware, J. H. Son, M. Cheng, R. V. Mishmash, J. Alicea, and B. Bauer, Ising Anyons in Frustration-Free MajoranaDimer Models, Phys. Rev. B 94, 115127 (2016).

[69] R. Z. Goldstein and E. C. Turner, A Formula for StiefelWhitney Homology Classes, Proc. Am. Math. Soc. 58, 339 (1976).

[70] Note that the barycentric subdivision triangulation illustrated in Ref. [47] is a special case of this general construction of the Stiefel-Whitney homology class [69].

[71] U. Pachner, P. L. Homeomorphic Manifolds Are Equivalent by Elementary Shellings, Eur. J. Combinatorics 12, 129 (1991).

[72] See Supplemental Material at http://link.aps.org/ supplemental/10.1103/PhysRevX.8.011055 for the physical origin of purely imaginary phase factor in obstruction, all Pachner moves and group structure of group supercohomology solutions.

[73] F. Costantino, A Calculus for Branched Spines of 3-Manifolds, Math. Z. 251, 427 (2005).

[74] If we choose the convention of pairing $\langle\overline{0} \overline{3}\rangle$ and $\langle\overline{1} \overline{2}\rangle$ of the four Majorana fermions at the center of tetrahedron $\langle 0123\rangle$, all of the results are the same as those of the conventional $\langle\overline{0} \overline{2}\rangle$ and $\langle\overline{1} \overline{3}\rangle$ used in the main text of our paper. $\gamma_{012 A}$ and $\gamma_{234 B}$ can also be chosen consistently in the definition of $X$ operators [see the discussion below Eq. (44)]. The last choice of pairing $\langle\overline{0} \overline{1}\rangle$ and $\langle\overline{2} \overline{3}\rangle$ is different from the above two conventions because the Majorana fermion parity is changed when four Kitaev strings meet at one tetrahedron. However, we can shift the $c$ fermion number $n_{3}\left(g_{0}, g_{1}, g_{2}, g_{3}\right)$ by one $(\bmod 2)$ to absorb this fermion parity change and then obtain the same obstruction results. 
[75] N. E. Steenrod, Products of Cocycles and Extensions of Mappings, Ann. Math. 48, 290 (1947).

[76] G. Brumfiel and J. Morgan (unpublished).

[77] M. Cheng, N. Tantivasadakarn, and C. Wang, Loop Braiding Statistics and Interacting Fermionic Symmetry-
Protected Topological Phases in Three Dimensions, preceding Letter, Phys. Rev. X 8, 011054 (2018).

[78] A. Kapustin and R. Thorngren, Fermionic SPT Phases in Higher Dimensions and Bosonization, J. High Energy Phys. 10 (2017) 080. 